\title{
The weakest failure detectors to boost obstruction-freedom
}

\author{
Rachid Guerraoui · Michał Kapałka • \\ Petr Kouznetsov
}

Received: 30 December 2006 / Accepted: 4 October 2007 / Published online: 31 October 2007

(C) Springer-Verlag 2007

\begin{abstract}
It is considered good practice in concurrent computing to devise shared object implementations that ensure a minimal obstruction-free progress property and delegate the task of boosting liveness to independent generic oracles called contention managers. This paper determines necessary and sufficient conditions to implement wait-free and non-blocking contention managers, i.e., contention managers that ensure wait-freedom (resp. non-blockingness) of any associated obstruction-free object implementation. The necessary conditions hold even when universal objects (like compare-and-swap) or random oracles are available in the implementation of the contention manager. On the other hand, the sufficient conditions assume only basic read/write objects, i.e., registers. We show that failure detector $\diamond \mathscr{P}$ is the weakest to convert any obstruction-free algorithm into a wait-free one, and $\Omega^{*}$, a new failure detector which we introduce in this paper, and which is strictly weaker than $\diamond \mathscr{P}$ but strictly stronger than $\Omega$, is the weakest to convert any obstruction-free algorithm into a non-blocking one. We also address the issue of minimizing the overhead imposed by contention management in low contention scenarios. We
\end{abstract}

This paper is a revised and extended version of a paper with the same title in the Proceedings of the 20th International Symposium on Distributed Computing (DISC'06).

R. Guerraoui · M. Kapałka ( $\square)$

School of Computer and Communication Sciences,

EPFL, Lausanne, Switzerland

e-mail: michal.kapalka@epfl.ch

R. Guerraoui

Computer Science and Artificial Intelligence Laboratory,

MIT, Cambridge, USA

P. Kouznetsov

Max Planck Institute for Software Systems,

Saarbrucken, Germany propose two intermittent failure detectors $I_{\Omega^{*}}$ and $I_{\diamond \mathscr{P}}$ that are in a precise sense equivalent to, respectively, $\Omega^{*}$ and $\diamond \mathscr{P}$, but allow for reducing the cost of failure detection in eventually synchronous systems when there is little contention. We present two contention managers: a non-blocking one and a wait-free one, that use, respectively, $I_{\Omega^{*}}$ and $I_{\diamond \mathscr{P}}$. When there is no contention, the first induces very little overhead whereas the second induces some non-trivial overhead. We show that wait-free contention managers, unlike their nonblocking counterparts, impose an inherent non-trivial overhead even in contention-free executions.

Keywords Shared memory - Obstruction-free . Non-blocking · Wait-free · Contention manager . Failure detector

\section{Introduction}

Multiprocessor systems are becoming more and more common nowadays. Multithreading thus becomes the norm and studying scalable and efficient synchronization methods is essential. Traditional locking-based techniques do not scale and may induce priority inversion, deadlock and fault-tolerance issues when a large number of threads is involved.

Wait-free synchronization algorithms [18] circumvent the issues of locking and guarantee individual progress even in presence of high contention. Wait-freedom is a liveness property which stipulates that every process completes every operation in a finite number of its own steps, regardless of the status of other processes, i.e., contending or even crashed. Ideal synchronization algorithms combine wait-freedom with linearizability $[3,21]$, a safety property which provides the illusion of instantaneous operation executions. 


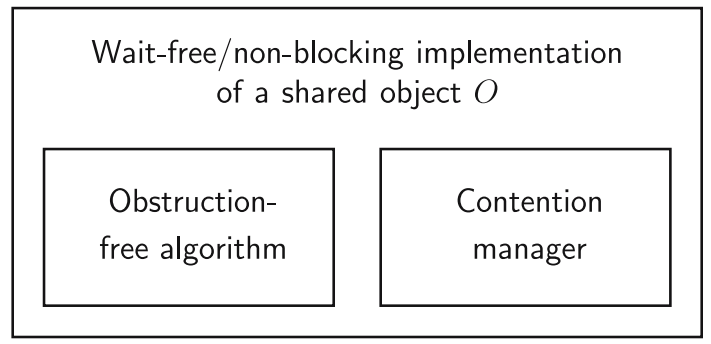

Fig. 1 A modular implementation of a shared object $O$

Alternatively, a liveness property called non-blockingness ${ }^{1}$ may be considered instead of wait-freedom. Non-blockingness guarantees global progress, i.e., that some process will complete an operation in a finite number of steps, regardless of the behaviour of other processes. Non-blockingness is weaker than wait-freedom as it might not protect some processes from starvation.

\subsection{Obstruction-freedom and contention managers}

Wait-free and non-blocking algorithms are, however, notoriously difficult to design $[4,23]$, especially with the practical goal to be fast in low contention scenarios, which are usually considered the most common in practice. An appealing principle to reduce this difficulty consists in separating two concerns of a synchronization algorithm: (1) ensuring linearizability with a weak conditional progress guarantee, and (2) boosting progress. More specifically, the idea is to focus on algorithms that ensure linearizability together with a weak liveness property called obstruction-freedom [20], and then combine these algorithms with separate generic oracles that boost progress, called contention managers $[15,19$, 27,28] (see Fig. 1). This separation lies at the heart of modern (obstruction-free) software transactional memory (STM) frameworks [19]. The approach simplifies the task of programmers, for they do not have to care about wait-freedom or non-blockingness. Instead they can focus on safety properties of their implementation, knowing that liveness can be boosted later, using a generic contention manager, possibly developed by concurrency experts and optimized for a given system.

An obstruction-free (or OF, for short) algorithm ensures progress only for processes that execute in isolation for sufficiently long time. In presence of high contention, however, OF algorithms can livelock, preventing any process from terminating. Contention managers are used precisely to cope with high contention scenarios. When queried by a process

\footnotetext{
1 The term non-blocking is defined here in the traditional way [18]: "some process will complete its operation in a finite number of steps, regardless of the relative execution speeds of the processes." This term is sometimes confused with the term lock-free.
}

executing an $\mathrm{OF}$ algorithm, a contention manager can delay the process for some time in order to boost the progress of other processes. The contention manager can neither share objects with the OF algorithm, nor return results on its behalf. If it did, the contention manager could peril the safety of the OF algorithm, hampering the overall separation of concerns principle.

In short, the goal of a contention manager is to provide processes with enough time without contention so that they can complete their operations. In its simplest form, a contention manager can be a randomized back-off protocol. More sophisticated contention management strategies have been experimented in practice $[16,27,28]$. Precisely because they are entirely devoted to progress, they can be combined or changed on the fly [15]. Most previous strategies were pragmatic, with no aim to provide worst case guarantees. In this paper we focus on contention managers that provide such guarantees. More specifically, we study contention managers that convert any OF algorithm into a non-blocking or wait-free one, and which we call, respectively, non-blocking or wait-free contention managers.

\subsection{Contention management and failure detectors}

Two wait-free contention managers have recently been proposed $[10,14]$. Both rely on timing assumptions to detect processes that fail in the middle of their operations. (The notion of failure might for instance model the fact that a process is swapped-out by the operating system for a long period.) This suggests that some information about failures might inherently be needed by any wait-free contention manager. But this is not entirely clear because, in principle, a contention manager could also use randomization to schedule processes, or even powerful synchronization primitives like compare-andswap, which is known to be universal, i.e., able to wait-free implement any other object [18]. In the parlance of [7], we would like to determine whether a failure detector is actually needed to implement a contention manager that provides strong liveness guarantees even in the worst case, and if it is, what is the weakest one [6]. Besides the theoretical interest, determining the weakest failure detector $\mathscr{D}$ for a given contention manager $C$ is, we believe, of practical relevance, for it provides a uniform implementation of $C$ in any system where $\mathscr{D}$ (and thus $C$ ) can actually be implemented.

A failure detector is a distributed oracle that periodically outputs, at each process, some information about which processes are still alive and which have already crashed (failed). Failure detectors differ in the quality of information they provide. For example, perfect failure detector $\mathscr{P}$ [7] ensures, intuitively, that every failure is eventually detected by every correct (i.e., non-faulty) process and that there is never any false detection. On the other hand, eventually perfect failure detector $\diamond \mathscr{P}$ [7] gives the same guarantees as $\mathscr{P}$ but 
only after some unknown, but finite, time. That is, the output of $\diamond \mathscr{P}$ can be arbitrary for any finite period of time, but eventually it stabilizes and becomes as accurate as for $\mathscr{P}$. The common property of $\mathscr{P}$ and $\diamond \mathscr{P}$ is that they provide each alive process with some information about the status of every other process. However, this is not always the case. For example, failure detector $\Omega$ [6] provides processes with a leadership information: it guarantees that eventually all correct processes will elect the same correct process as their leader.

Clearly, any output of $\mathscr{P}$ is also a valid output of $\diamond \mathscr{P}$. Thus, having $\mathscr{P}$ in a system, we can also have $\diamond \mathscr{P}$ at no cost. It is also straightforward to implement $\Omega$ in a system that already has $\mathscr{P}$ : every process simply chooses the noncrashed (according to $\mathscr{P}$ ) process with the lowest id as its leader. In fact, if we used $\diamond \mathscr{P}$ instead of $\mathscr{P}$ in this algorithm, we would still have a correct implementation of $\Omega$. On the contrary, if we only have $\Omega$ in a system, we can implement neither $\mathscr{P}$ nor $\diamond \mathscr{P}$. Also, it is impossible to transform the output of $\diamond \mathscr{P}$ into a valid output of $\mathscr{P}$ in an asynchronous system.

The above discussion highlights a way in which failure detectors can be compared. Informally, a failure detector $\mathscr{D}$ is said to be weaker than a failure detector $\mathscr{D}^{\prime}$ if we can implement $\mathscr{D}$ using $\mathscr{D}^{\prime}$ in an asynchronous system [6]. If the opposite is not true, i.e., if we cannot implement $\mathscr{D}^{\prime}$ out of $\mathscr{D}$, then $\mathscr{D}$ is said to be strictly weaker than $\mathscr{D}^{\prime}$.

\subsection{Minimal failure detectors to boost obstruction-freedom}

We show in this paper that the eventually perfect failure detector $\diamond \mathscr{P}$ is the weakest to implement a wait-free contention manager. We also introduce a failure detector $\Omega^{*}$, which we show is the weakest to implement a non-blocking contention manager. Failure detector $\Omega^{*}$ is strictly weaker than $\diamond \mathscr{P}$, and strictly stronger than failure detector $\Omega$, known to be the weakest to wait-free implement the (universal) consensus object [6].

It might look surprising that $\Omega$ is not sufficient to implement a wait-free or even a non-blocking contention manager. For example, the seminal Paxos algorithm [24] uses $\Omega$ to transform an OF implementation of consensus into a waitfree one [5]. Each process that is eventually elected leader by $\Omega$ is given enough time to run alone, reach a decision and communicate it to the others. This approach does not help, however, if we want to make sure that processes make progress regardless of the actual (possibly long-lived) object and its OF implementation. Intuitively, the leader elected by $\Omega$ may have no operation to perform while other processes may livelock forever. Because a contention manager cannot make processes help each other, the output of $\Omega$ is not sufficient: this is so even if randomized oracles or universal objects are available. Intuitively, wait-free contention managers need a failure detector that would take care of every non-crashed process with a pending operation so that the process can run alone for sufficiently long time. As for non-blocking contention managers, at least one correct process with a pending operation should be given enough time to run alone.

To prove each of the weakest failure detector results, we first present (necessary part) a reduction algorithm [6] that extracts the output of failure detector $\Omega^{*}$ (respectively, $\diamond \mathscr{P}$ ) using a non-blocking (respectively, wait-free) contention manager implementation. When devising our reduction algorithms, we do not restrict what objects (or random oracles) can be used by the contention manager or the OF algorithm. Then (sufficient part) we present algorithms that implement the contention managers using the failure detectors and simple register objects.

It is worthwhile noticing that proving the results goes through defining the notions of non-blocking and wait-free contention managers and specifying the interactions between OF algorithms and contention managers. These, we believe, are interesting contributions in their own right.

\subsection{Reducing the cost of contention management}

Our implementations of contention managers use failure detectors $\Omega^{*}$ and $\diamond \mathscr{P}$. In some systems it is possible to implement $\Omega^{*}$ and $\diamond \mathscr{P}$ efficiently, e.g., when there is some failure detection functionality in the operating system [4]. In general, however, when timeout-based mechanisms have to be used, this is not the case. The problem with failure detectors in their conventional form [7] is that their output cannot depend on computations being performed by processes. Thus, a timeout-based implementation of $\Omega^{*}$ (and a fortiori of $\diamond \mathscr{P}$ ) will have to make processes exchange "heartbeat" signals even when failure detection is not actually needed. For example, in executions with low contention between processes, a failure detector might not be necessary at all, and a simple contention manager using, for example, an exponential back-off scheme would be sufficient to provide progress. Ideally we would like a failure detection mechanism to be involved only when needed.

To cope with this issue, we introduce the notion of an intermittent failure detector (IFD). Although an IFD is not a failure detector in the sense of [7], it gives processes some information about failures. There are however two important specificities of an IFD. First, its modules, running at different processes, can be stopped and restarted at any time independently. Thus, a process that does not need any information about failures simply stops its local IFD module. Second, intermittent failure detectors return only information about failures a process explicitly queried for, similarly to [9]. This enables frugal IFD implementations.

We present two intermittent failure detectors, $I_{\Omega} *$ and $I_{\diamond \mathscr{P}}$, and example implementations of theirs in eventually 
synchronous systems. We establish a formal relationship between a failure detector $\mathscr{D}$ and its intermittent variant $I_{\mathscr{D}}$, by proving that the latter provides as much information about failures as the former. We do so by treating $I \mathscr{D}$ as an abstract problem and proving that $\mathscr{D}$ is the weakest failure detector to implement $I_{\mathscr{D}}$. Intuitively, in the worst case (in terms of contention), $I_{\Omega^{*}}$ and $I_{\diamond \mathscr{P}}$ give processes the same amount of information about failures as, respectively, $\Omega^{*}$ or $\diamond \mathscr{P}$. However, in many scenarios intermittent failure detectors can be used in a more efficient way than their failure detector counterparts. Namely, a process triggers a failure detection mechanism only when the process needs some information about failures. Clearly, this may cause failures to be detected with a much larger delay than for classical failure detectors. However, in the arguably most common scenarios of low contention and low failure rate, intermittent failure detection is appealing.

We present a non-blocking contention manager $C M_{\mathrm{nb}}$ and a wait-free contention manager $C M_{\mathrm{wf}}$ that use intermittent failure detectors $I_{\Omega^{*}}$ and $I_{\diamond \mathscr{P}}$, respectively. Both contention managers can be easily combined with heuristic contention management strategies $[27,28]$ to achieve good average-case performance. Also, both are minimal in terms of failure information. In executions with no contention, i.e., when processes always run alone, contention manager $C M_{\mathrm{nb}}$ imposes very little overhead: its implementation (together with the underlying implementation of IFD $I_{\Omega^{*}}$ ) does not require any communication between processes. On the contrary, contention manager $C M_{\mathrm{wf}}$ provides some level of overhead which we prove is unavoidable. While doing so, we exhibit an interesting "overhead gap" between non-blocking and wait-free contention management.

\subsection{Related work}

Obstruction-freedom, as a weak liveness property, was introduced by Herlihy et al. [20]. They proposed to delegate stronger progress guarantees to specialized contention management oracles. In this paper, we present implementations of contention managers that ensure progress (non-blockingness or wait-freedom) when combined with any obstruction-free algorithm.

Our contention manager implementations share many similarities with the algorithms of [10] and [14], both of which ensure wait-freedom, but use timeout-based failure detection mechanisms directly. In fact, the techniques used in all these algorithms originate in indulgent algorithms [13] designed for partially synchronous systems $[7,8,24]$, ported later to shared memory systems $[5,12]$. However, the way our contention managers obtain information about failures-from (intermittent) failure detectors - and the way they can be combined with heuristic contention management techniques are, we believe, novel. The implementations of IFDs $I_{\Omega} *$ and $I_{\diamond \mathscr{P}}$ we give in this paper are similar to known message passing implementations of $\diamond \mathscr{P}[1,7,9,25]$.

\subsection{Roadmap}

The paper is organized as follows. Section 2 presents our system model and formally defines wait-free and non-blocking contention managers. In Sects. 3 and 4, we prove our weakest failure detector results. Then, in Sect. 5, we introduce the abstraction of an intermittent failure detector (IFD) and

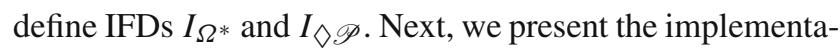
tions of contention managers $C M_{\mathrm{nb}}$ and $C M_{\mathrm{wf}}$ (Sect. 6) and example implementations of $I_{\Omega} *$ and $I_{\diamond \mathscr{P}}$ in eventually synchronous systems (Sect. 7). In Sect. 8, we discuss the overhead of non-blocking and wait-free contention managers. We conclude the paper with some final remarks in Sect. 9.

\section{Preliminaries}

\subsection{Processes and failure detectors}

We consider a set of $n$ processes $\Pi=\left\{p_{1}, \ldots, p_{n}\right\}$ in a shared memory system $[18,22]$. A process executes the (possibly randomized) algorithm assigned to it, until the process crashes (fails) and stops executing any action. We assume the existence of a global discrete clock that is, however, inaccessible to the processes. We say that a process is correct if it never crashes. We say that process $p_{i}$ is alive at time $t$ if $p_{i}$ has not crashed by time $t$.

A failure detector $[6,7]$ is a distributed oracle that provides every process with some information about failures. The output of a failure detector depends only on which and when processes fail, and not on computations being performed by the processes. A process $p_{i}$ queries a failure detector $\mathscr{D}$ by accessing local variable $\mathscr{D}$-output - $_{i}$ the output of the module of $\mathscr{D}$ at process $p_{i}$. Failure detectors can be partially ordered according to the amount of information about failures they provide. A failure detector $\mathscr{D}$ is weaker than a failure detector $\mathscr{D}^{\prime}$, and we write $\mathscr{D} \preceq \mathscr{D}^{\prime}$, if there is an algorithm (called a reduction algorithm) that uses $\mathscr{D}^{\prime}$ (as the only source of information about failures) to emulate the output of $\mathscr{D}$ [6]. If $\mathscr{D} \preceq \mathscr{D}^{\prime}$ but $\mathscr{D}^{\prime} \npreceq \mathscr{D}$, we say that $\mathscr{D}$ is strictly weaker than $\mathscr{D}^{\prime}$, and we write $\mathscr{D} \prec \mathscr{D}^{\prime}$.

\subsection{Base and high-level objects}

Processes communicate by invoking primitive operations (which we will call instructions) on base shared objects and seek to implement the operations of a high-level shared object $O$. Object $O$ is in turn used by an application, as a high-level inter-process communication mechanism. We call invocation and response events of a high-level operation op on the 
implemented object $O$ application events and denote them by, respectively, inv (op) and ret (op) (or $i n v_{i}(o p)$ and $r e t_{i}(o p)$ at a process $p_{i}$ ).

An implementation of $O$ is a distributed algorithm that specifies, for every process $p_{i}$ and every operation $o p$ of $O$, the sequences of steps that $p_{i}$ should take in order to complete $o p$. Process $p_{i}$ completes operation $o p$ when $p_{i}$ returns from $o p$. Every process $p_{i}$ may complete any number of operations but, at any point in time, at most one operation op can be pending (started and not yet completed) at $p_{i}$.

We consider implementations of $O$ that combine a subprotocol that ensures safety and a weak liveness property, called obstruction-freedom, with a sub-protocol that boosts this liveness guarantee. The former is called an obstructionfree $(O F)$ algorithm $A$ and the latter a contention manager $C M$. We focus on linearizable $[3,21]$ implementations of $O$ : every operation appears to the application as if it took effect instantaneously between its invocation and its return. An implementation of $O$ involves two categories of steps executed by any process $p_{i}$ : those (executed on behalf) of $C M$ and those (executed on behalf) of $A$. In each step, a process $p_{i}$ either executes an instruction on a base shared object or queries a failure detector. The latter case occurs only if $p_{i}$ executes a step on behalf of $C M$.

Obstruction-freedom $[19,20]$ stipulates that if a process invokes an operation $o p$ on object $O$ and from some point in time executes steps of $A$ alone, ${ }^{2}$ then the process eventually completes $o p$. Non-blockingness stipulates that if some correct process never completes an invoked operation, then some other process completes infinitely many operations. Wait-freedom [18] is stronger and ensures that every correct process that invokes an operation eventually returns from the operation.

\subsection{Interaction between modules}

OF algorithm $A$, executed by any process $p_{i}$, communicates with contention manager $C M$ via calls try $y_{i}$ and resign $n_{i}$ implemented by $C M$ (see Fig. 2). Process $p_{i}$ invokes $t r y_{i}$ just after $p_{i}$ starts an operation, and also later (even several times before $p_{i}$ completes the operation) to signal possible contention. Process $p_{i}$ invokes resign $n_{i}$ just before returning from an operation, and always eventually returns from this call (or crashes). Both calls, try $_{i}$ and resign $n_{i}$, return $o k$.

An example OF algorithm that uses this model of interaction with a contention manager is Algorithm 1. The algorithm implements a timestamping mechanism and is based on the implementation of a splitter [26]. It is not meant to be practical or efficient-it just shows how calls try and resign should be used.

$\overline{2}$ That is without encountering step contention [2].

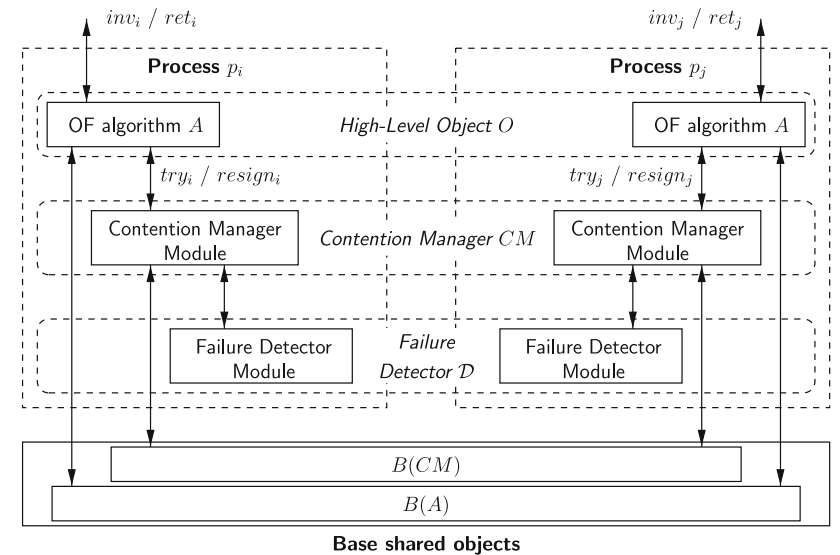

Fig. 2 The OF algorithm/contention manager interface

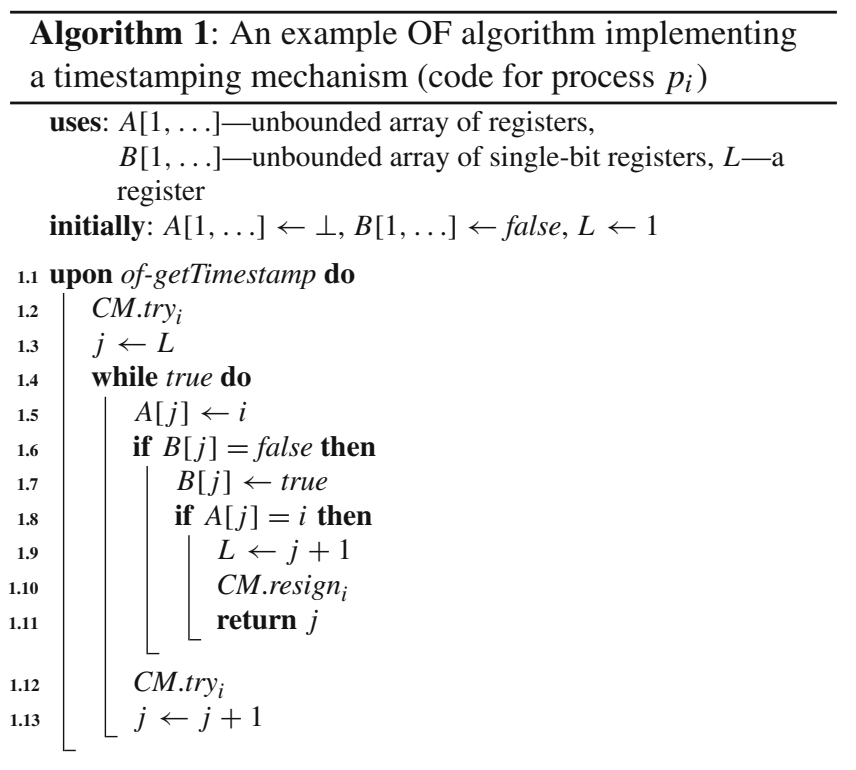

The intuition behind the algorithm is the following. A process $p_{i}$ that invokes of-getTimestamp scans the array $B$ of registers, starting from the index stored in register $L$, to find the lowest value $j$ for which $B[j]$ is false. Then, $p_{i}$ sets $B[j]$ to true. (The value of $B[j]$ will never change thereafter.) If no other process is executing steps concurrently to $p_{i}$, then $p_{i}$ returns $j$ as a new timestamp. Also, $p_{i}$ stores value $j+1$ in register $L$ to optimize future invocations of of-getTimestamp. The splitter code in lines 1.5-1.8 guarantees that at most one process can return a given value $j$, thus ensuring that the timestamps are unique. However, if two or more processes execute the algorithm concurrently, it might happen that none of them ever returns. That is why it is important that a contention manager delays some processes and let only one execute steps of the algorithm at a time.

We denote by $B(A)$ and $B(C M)$ the sets of base shared objects, always disjoint, that can be possibly accessed by steps of, respectively, $A$ and $C M$, in every execution, by every 
process. Calls try and resign are thus the only means by which $A$ and $C M$ interact. The events corresponding to invocations of, and responses from, try and resign are called cm-events. We denote by $t r y_{i}^{\text {inv }}$ and resign $n_{i}^{\text {inv }}$ an invocation of call try and resign $_{i}$, respectively (at process $p_{i}$ ), and by $\operatorname{tr} y_{i}^{\text {ret }}$ and

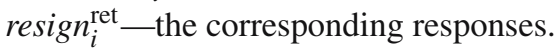

\subsection{Executions and histories}

An execution of an OF algorithm $A$ combined with a contention manager $C M$ is a sequence of events that include steps of $A$, steps of $C M, \mathrm{~cm}$-events and application events. Every event in an execution is associated with a unique time representing the moment at which the event took place. Simultaneous events (say in case of multiprocessors) are arbitrarily ordered. Every execution $e$ induces a history $H(e)$ that includes only application events (invocations and responses of high-level operations). The corresponding CM-history $H_{\mathrm{CM}}(e)$ is the longest subsequence of $e$ containing only application events and cm-events of the execution, and the corresponding $O F$-history $H_{\mathrm{OF}}(e)$ is the longest subsequence of $e$ containing only application events, cm-events, and steps of $A$. For a sequence $s$ of events, $s \mid i$ denotes the longest subsequence of $s$ containing only events at process $p_{i}$.

We say that a process $p_{i}$ is blocked at time $t$ in an execution $e$ if (1) $p_{i}$ is alive at time $t$, and (2) the latest event in $H_{\mathrm{CM}}(e) \mid i$ that occurred before $t$ is $t r y_{i}^{\text {inv }}$ or resign $n_{i}^{\text {inv }}$. A process $p_{i}$ is busy at time $t$ in $e$ if (1) $p_{i}$ is alive at time $t$, and (2) the latest event in $H_{\mathrm{CM}}(e) \mid i$ that occurred before $t$ is $t r y_{i}^{\mathrm{ret}}$. We say that a process $p_{i}$ is active at $t$ in $e$ if $p_{i}$ is either busy or blocked at time $t$ in $e$. We say that a process $p_{i}$ is idle at time $t$ in $e$ if $p_{i}$ is not active at $t$ in $e .^{3}$ A process resigns when it invokes resign on a contention manager.

We say that a process $p_{i}$ is obstruction-free in an interval $\left[t, t^{\prime}\right]$ in an execution $e$, if $p_{i}$ is the only process that takes steps of $A$ in $\left[t, t^{\prime}\right]$ in $e$ and $p_{i}$ is not blocked infinitely long in $\left[t, t^{\prime}\right]$ (if $t^{\prime}=\infty$ ). We say that process $p_{i}$ is eventually obstruction-free at time $t$ in $e$ if $p_{i}$ is active at $t$ or later and $p_{i}$ either resigns after $t$ or is obstruction-free in the interval $\left[t^{\prime}, \infty\right)$ for some $t^{\prime}>t$. Note that, since algorithm $A$ is obstruction-free, if a correct active process $p_{i}$ is eventually obstruction-free at some point in time, then $p_{i}$ eventually resigns and completes its operation thereafter.

\subsection{Well-formed executions}

We impose certain restrictions on the way an $\mathrm{OF}$ algorithm $A$ and a contention manager $C M$ interact. In particular, we assume that no process takes steps of $A$ while being blocked by $C M$ or idle, and no process takes infinitely many steps of $A$ without calling $C M$ infinitely many times. Further, a process

\footnotetext{
${ }^{3}$ Note that every process that has crashed is permanently idle.
}

must inform $C M$ that an operation is completed by calling resign before returning the response to the application.

Formally, we assume that every execution $e$ is well-formed, i.e., $H(e)$ is linearizable $[3,21]$, and, for every process $p_{i},(1)$ $H_{\mathrm{CM}}(e) \mid i$ is a prefix of a sequence $\left[o p_{1}\right]\left[o p_{2}\right], \ldots$, where each $\left[o p_{k}\right]$ has the form $i n v_{i}\left(o p_{k}\right), t r y_{i}^{\text {inv }}, t r y_{i}^{\text {ret }}, \ldots, \operatorname{tr} y_{i}^{\text {inv }}$, $\operatorname{try}_{i}^{\mathrm{ret}}, \operatorname{resign}_{i}^{\mathrm{inv}}, \operatorname{resign}_{i}^{\mathrm{ret}}, \operatorname{ret}_{i}\left(\mathrm{op}_{k}\right)$; (2) in $H_{\mathrm{OF}}(e) \mid i$, no step of $A$ is executed when $p_{i}$ is blocked or idle, (3) in $H_{\mathrm{OF}}(e) \mid i$, $i n v_{i}$ can only be followed by $t r y_{i}^{\text {inv }}$, and $r e t_{i}$ can only be preceded by resign $n_{i}^{\text {ret }}$ (4) if $p_{i}$ is busy at time $t$ in $e$, then at some $t^{\prime}>t$, process $p_{i}$ is idle or blocked. The last condition implies that every busy process $p_{i}$ eventually invokes $\operatorname{try}_{i}$ (and becomes blocked), resigns or crashes. Clearly, in a well-formed execution, every process goes through the following cyclical order of modes: idle, active, idle, ..., where each active period consists itself of a sequence blocked, busy, blocked,....

\subsection{Non-blocking contention manager}

We say that a contention manager CM guarantees non-blockingness for an OF algorithm $A$ if in each execution $e$ of $A$ combined with $C M$ the following property is satisfied: if some correct process is active at a time $t$, then at some time $t^{\prime}>t$ some process resigns.

We say that a contention manager $C M$ is non-blocking if, for every OF algorithm $A$, in every execution of $A$ combined with $C M$ the following property is ensured at every time $t$ :

Global Progress. If some correct process is active at $t$, then some correct process is eventually obstruction-free at $t$.

Intuitively, a non-blocking contention manager allows at least one active process to be obstruction-free (and busy) for sufficiently long time, so that the process can complete its operation.

Theorem 1 A contention manager CM guarantees nonblockingness for every $O F$ algorithm if and only if $C M$ is non-blocking.

Proof $(\Rightarrow)$ Consider a contention manager $C M$ that guarantees non-blockingness for every OF algorithm. Let $A$ be any $\mathrm{OF}$ algorithm and $e$ be any execution of $A$ combined with $C M$. Let some correct process be active at time $t$ in $e$. Since $C M$ guarantees non-blockingness, some active process resigns at some future time, and the Global Progress property is trivially ensured.

$(\Leftarrow)$ By contradiction, assume that there exists a nonblocking contention manager $C M$ such that, for some $\mathrm{OF}$ algorithm $A$, there is an execution $e$ of $A$ combined with $C M$, such that some correct process is active at $t$, and no active process resigns after $t$. By Global Progress, some correct active process $p_{i}$ is eventually obstruction-free at $t$. Since 
$A$ is obstruction-free and $p_{i}$ takes infinitely many steps of $A$ in isolation, $p_{i}$ must complete its operation and resign after $t$ - a contradiction.

\subsection{Wait-free contention manager}

We say that a contention manager $C M$ guarantees wait-freedom for an OF algorithm $A$ if in every execution $e$ of $A$ combined with $C M$ the following property is satisfied: if a process $p_{i}$ is active at a time $t$, then at some time $t^{\prime}>t, p_{i}$ becomes idle. In other words, every operation executed by a correct process eventually returns.

A contention manager $C M$ is wait-free if, for every $\mathrm{OF}$ algorithm $A$, in every execution of $A$ combined with $C M$, the following property is ensured at every time $t:{ }^{4}$

Fairness. If a correct process $p_{i}$ is active at $t$, then $p_{i}$ is eventually obstruction-free at $t$.

Intuitively, a wait-free contention manager makes sure that every correct active process is given "enough" time to complete its operation, regardless of how other processes behave.

Theorem 2 A contention manager CM guarantees waitfreedom for every $O F$ algorithm if and only if $C M$ is wait-free.

Proof $(\Rightarrow)$ Consider a contention manager $C M$ that guarantees wait-freedom for every $\mathrm{OF}$ algorithm. Let $A$ be any $\mathrm{OF}$ algorithm and $e$ be any execution of $A$ combined with $C M$. Since in $e$ every active process is eventually idle, every correct active process eventually resigns in $e$, and so the Fairness property is trivially satisfied.

$(\Leftarrow)$ Let $C M$ be a wait-free contention manager, and $A$ be any $\mathrm{OF}$ algorithm. Consider any execution $e$ of $A$ combined with $C M$.

Suppose, by contradiction, that some correct process $p_{i}$ is active at time $t$ and never completes its operation thereafter. But then, by Fairness, $p_{i}$ is eventually obstruction-free at $t$ and so $p_{i}$ is obstruction-free in period $\left[t^{\prime}, \infty\right)$ for some $t^{\prime}>t$. Therefore, since $A$ is obstruction-free and $p_{i}$ takes infinitely many steps of $A$ in isolation, $p_{i}$ must eventually resign and complete its operation-a contradiction.

In the following, we seek to determine the weakest [6] failure detector $\mathscr{D}$ to implement a non-blocking (resp. wait-free) contention manager $C M$. This means that (1) $\mathscr{D}$ implements such a contention manager, i.e., there is an algorithm that implements $C M$ using $\mathscr{D}$, and (2) $\mathscr{D}$ is necessary to implement such a contention manager, i.e., if a failure detector $\mathscr{D}^{\prime}$ implements $C M$, then $\mathscr{D} \preceq \mathscr{D}^{\prime}$. In our context, a reduction algorithm that transforms $\mathscr{D}^{\prime}$ into $\mathscr{D}$ uses the $\mathscr{D}^{\prime}$-based

\footnotetext{
$\overline{4}$ This property is ensured by wait-free contention managers from the literature $[10,14]$.
}

implementation of the corresponding contention manager as a "black box" and read-write registers.

\section{Non-blocking contention managers}

\subsection{Failure detector $\Omega^{*}$}

Let $S \subseteq \Pi$ be a non-empty set of processes. Failure detector $\Omega_{S}$ outputs, at every process, an identifier of a process (called a leader), such that all correct processes in $S$ eventually agree on the identifier of the same correct process in $S .{ }^{5}$

Failure detector $\Omega^{*}$ is the composition $\left\{\Omega_{S}\right\}_{S \subset \Pi, S \neq \emptyset}$ : at every process $p_{i}, \Omega^{*}$-output $t_{i}$ is a tuple consisting of the outputs of failure detectors $\Omega_{S}$. We position $\Omega^{*}$ in the hierarchy of failure detectors of [7] by proving the following theorem:

Theorem $3 \Omega \prec \Omega^{*} \prec \diamond \mathscr{P}$.

Proof It is immediate that $\Omega$ is weaker than $\Omega^{*}: \Omega_{\Pi}$ is the same as $\Omega$. In a system of three or more processes, $\Omega$ is strictly weaker than $\Omega^{*}$. Indeed, consider a system of three processes, $p_{1}, p_{2}$, and $p_{3}$, and assume, by contradiction, that $\Omega^{*}$ is weaker than $\Omega$, i.e., that there exists a reduction algorithm $T_{\Omega \rightarrow \Omega^{*}}$ which extracts the output of $\Omega^{*}$ using $\Omega$. Take an execution $e$ of $T_{\Omega \rightarrow \Omega^{*}}$ in which $p_{3}$ is correct, $p_{2}$ is faulty, $\Omega$ always outputs $p_{3}$ at every process and consider the emulated output of $\Omega_{\left\{p_{1}, p_{2}\right\}}$. Since $p_{1}$ is the only correct process in $\left\{p_{1}, p_{2}\right\}$, there is a finite prefix $e^{\prime}$ of $e$ in which $\Omega_{\left\{p_{1}, p_{2}\right\}}$ outputs $p_{1}$ at $p_{1}$. But this finite execution is indistinguishable from a finite execution $e^{\prime \prime}$ in which $p_{2}$ is correct but slow. Now consider a finite extension of $e^{\prime \prime}$ in which $p_{1}$ fails, and thus eventually $\Omega_{\left\{p_{1}, p_{2}\right\}}$ outputs $p_{2}$ at $p_{2}$. But this finite execution is indistinguishable from a finite execution in which $p_{1}$ is correct but slow. By repeating this argument, we obtain an infinite execution of $T_{\Omega \rightarrow \Omega^{*}}$ in which both $p_{1}$ and $p_{2}$ are correct, and the output $\Omega_{\left\{p_{1}, p_{2}\right\}}$ never stabilizes at a single correct process-a contradiction.

It is immediate that $\Omega^{*}$ is weaker than $\diamond \mathscr{P}$ : eventually each correct process $p_{i}$ has complete and accurate information from $\diamond \mathscr{P}$ about failures of all other processes, so $p_{i}$ can perform an eventually perfect leader election in each subset of processes $p_{i}$ belongs to, thus extracting the output of $\Omega^{*}$.

To show that $\Omega^{*}$ is strictly weaker than $\diamond \mathscr{P}$, consider a system of two processes, $p_{1}$ and $p_{2}$, and assume, by contradiction, that $\diamond \mathscr{P}$ is weaker than $\Omega^{*}$, i.e., that there exists a reduction algorithm $T_{\Omega^{*} \rightarrow \diamond \mathscr{P}}$ which extracts the output of $\diamond \mathscr{P}$ using $\Omega^{*}$.

\footnotetext{
$5 \Omega_{S}$ can be seen as a restriction of the eventual leader election failure detector $\Omega$ [6] to processes in $S$. The definition of $\Omega_{S}$ resembles the notion of $\Gamma$-accurate failure detectors introduced in [17]. Clearly, $\Omega_{\Pi}$ is $\Omega$.
} 


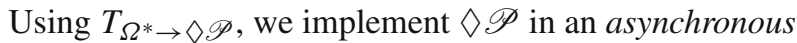
system, establishing a contradiction with $[7,11]$. In the implementation, the processes run two parallel algorithms, $T_{1}$ and

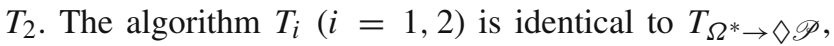
except that, instead of querying $\Omega^{*}$, it assumes that the $\Omega_{\left\{p_{1}, p_{2}\right\}}$ component of $\Omega^{*}$ always outputs $p_{i}$ at each process. (Clearly, $\Omega_{\left\{p_{i}\right\}}$ must always output $p_{i}$.) Note that every finite execution of $T_{i}$ is also a finite execution of $T_{\Omega^{*} \rightarrow \diamond \mathscr{P}}$. If $p_{i}$ is correct, then every (even infinite) execution of $T_{i}$ is also

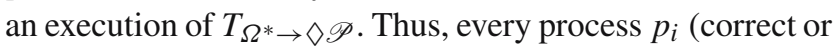
not) obtains from $T_{i}$ a valid output of $\diamond \mathscr{P}$. But $T_{i}$ does not use any failure detector, and so we get an implementation of $\diamond \mathscr{P}$ in an asynchronous system.

\subsection{The necessity part}

To show that $\Omega^{*}$ is necessary to implement a non-blocking contention manager, it suffices to prove that, for every nonempty $S \subseteq \Pi, \Omega_{S}$ is necessary to implement a non-blocking contention manager. Let $C M$ be a non-blocking contention manager using failure detector $\mathscr{D}$. We show that $\Omega^{*} \preceq \mathscr{D}$ by presenting an algorithm $T_{\mathscr{D} \rightarrow \Omega_{S}}$ (Algorithm 2) that, using $C M$ and $\mathscr{D}$, emulates the output of $\Omega_{S}$.

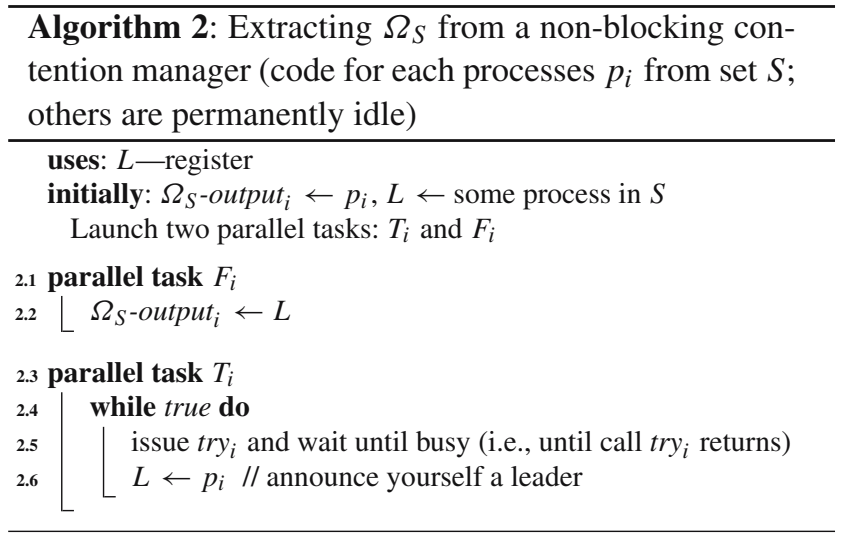

The algorithm works as follows. Every process $p_{i} \in S$ runs two parallel tasks $T_{i}$ and $F_{i}$. In task $T_{i}$, process $p_{i}$ periodically (1) gets blocked by $C M$ after invoking $\operatorname{try}_{i}$ (line 2.5), and (2) once $p_{i}$ gets busy again, announces itself a leader for set $S$ by writing its id in $L$ (line 2.6). In task $F_{i}$, process $p_{i}$ periodically determines its leader by reading register $L$ (line 2.2). ${ }^{6}$

Thus, no process ever resigns and every correct process in $S$ is permanently active from some point in time. Intuitively,

\footnotetext{
${ }^{6}$ If a process is blocked in one task, it continues executing steps in parallel tasks.
}

this signals a possible livelock to $C M$ which has to eventually block all active processes except for one that should run obstruction-free for sufficiently long time. By Global Progress, $C M$ cannot block all active processes forever, and so if the elected process crashes (and so becomes idle), $C M$ lets another active process run obstruction-free. Eventually, all correct processes in $S$ agree on the same correct process in $S$. Processes outside $S$ are permanently idle and permanently output their own ids: they do not access $C M$.

This approach contains a subtlety. To make sure that there is a time after which the same correct leader in $S$ is permanently elected by the correct processes in $S$, we do not allow the elected leader to resign (the output of $\Omega_{S}$ has to be eventually stable). This violates the assumption that processes using $C M$ run an obstruction-free algorithm, and thus, a priori, $C M$ is not obliged to preserve Global Progress. However, as we show below, since $C M$ does not "know" how much time a process executing an $\mathrm{OF}$ algorithm requires to complete its operation, $C M$ has to provide some correct process with unbounded time to run in isolation.

Theorem 4 Every non-blocking contention manager can be used to implement failure detector $\Omega^{*}$.

Proof Let $S \subseteq \Pi, S \neq \varnothing$ and consider any execution of Algorithm 2. If $S$ contains no correct process, then $\Omega_{S}$-output ${ }_{i}$ (for every process $p_{i} \in S$ ) trivially satisfies the property of $\Omega_{S}$. Now assume that there is a correct process in $S$. We claim that $C M$ eventually lets exactly one correct process in $S$ run obstruction-free while blocking forever all the other processes in $S$.

Suppose not. We obtain an execution in which every correct process in $S$ is allowed to be obstruction-free only for bounded periods of time. But the CM-history of this execution corresponds to an execution of some OF algorithm $A$ combined with $C M$ in which no active process ever completes its operation because no active process ever obtains enough time to run in isolation. Thus, no active process is eventually obstruction-free in that execution. This contradicts the assumption that $C M$ is non-blocking.

Therefore, there is a time after which exactly one correct process $p_{j} \in S$ is periodically busy (others are blocked or idle forever) and, respectively, register $L$ permanently stores the identifier of $p_{j}$. Thus, eventually, every correct process in $S$ outputs $p_{j}$ : the output of $\Omega_{S}$ is extracted.

\subsection{The sufficiency part}

We describe an implementation of a non-blocking contention manager using $\Omega^{*}$ and registers in Algorithm 3. The algorithm works as follows. All active processes, upon calling try, participate in the leader election mechanism using $\Omega^{*}$ in lines $3.3-3.5$. The active process $p_{i}$ that is elected a leader returns from try and is (eventually) allowed to run 
obstruction-free until $p_{i}$ resigns. Once $p_{i}$ resigns, the processes elect another leader. Failure detector $\Omega^{*}$ guarantees that if an active process is elected and crashes before resigning, another active process is eventually elected.

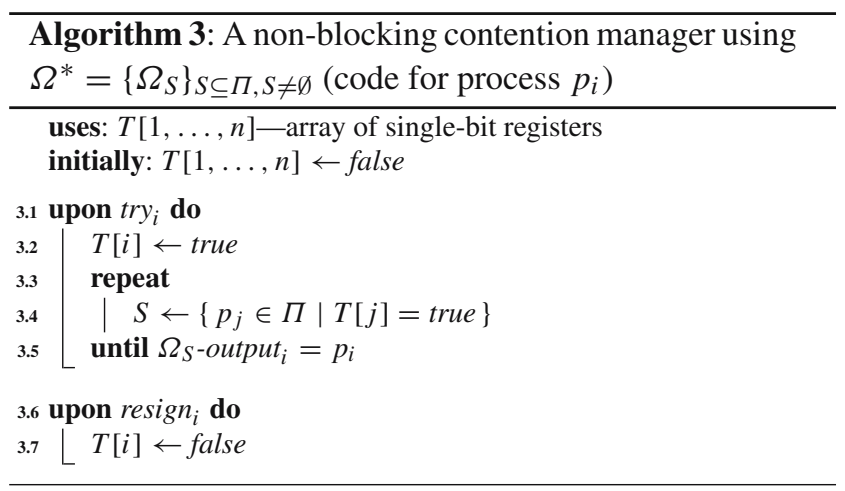

Lemma 1 The contention manager implemented by Algorithm 3 guarantees non-blockingness for every OF algorithm.

Proof Assume, by contradiction that there exists an OF algorithm $A$ for which contention manager $C M$ implemented by Algorithm 3 does not guarantee non-blockingness, i.e., there exists an execution $e$ of $A$ combined with $C M$ in which there are a correct process $p_{i}$ and a time $t$, such that $p_{i}$ is active at $t$ but for all $t^{\prime}>t$, no active process resigns at $t^{\prime}$.

Take any time $t^{\prime}>t$. Let us denote by $S\left(t^{\prime}\right)$ the set of all processes $p_{j}$ such that $T[j]=$ true at time $t^{\prime}$ in $e$. Since no active process resigns after $t$, there is a time $t^{*} \geq t$ and a set $S$, such that for all $t^{\prime}>t^{*}, S\left(t^{\prime}\right)=S$. By the algorithm, $p_{i}$ eventually sets $T[j]$ to true. Thus, $p_{i}$ is in $S$, i.e., $S$ includes at least one correct process. At every correct process in $S$, $\Omega_{S}$ eventually outputs the same correct process $p_{j}$ in set $S$ (a leader).

Since every active process eventually invokes try, resigns or crashes (by the properties of OF algorithms), and no process resigns after $t^{*}$, there is a time $t^{\prime}>t^{*}$ after which every correct process except for $p_{j}$ gets permanently blocked in lines 3.3-3.5. That is because $p_{j}$ does not resign after $t$ and so $p_{j}$ does not reset $T[j]$ to false thereafter and remains the leader for set $S$ forever. Thus, $p_{j}$ is eventually obstructionfree at $t$. Since $p_{j}$ runs an obstruction-free algorithm $A$, it eventually resigns and completes its operation-a contradiction.

From Theorem 1 and Lemma 1 we immediately obtain the following result:

Theorem 5 Algorithm 3 implements a non-blocking contention manager.

\section{Wait-free contention managers}

We prove here that the weakest failure detector to implement a wait-free contention manager is $\diamond \mathscr{P}$ [7]. Failure detector $\diamond \mathscr{P}$ outputs, at each time and every process, a set of suspected processes. There is a time after which (1) every crashed process is permanently suspected by every correct process and (2) no correct process is ever suspected by any correct process.

\subsection{The necessity part}

We first consider a wait-free contention manager $C M$ using a failure detector $\mathscr{D}$, and we exhibit a reduction algorithm $T_{\mathscr{D} \rightarrow \diamond \mathscr{P}}$ (Algorithm 4) that, using $C M$ and $\mathscr{D}$, emulates the output of $\diamond \mathscr{P}$.

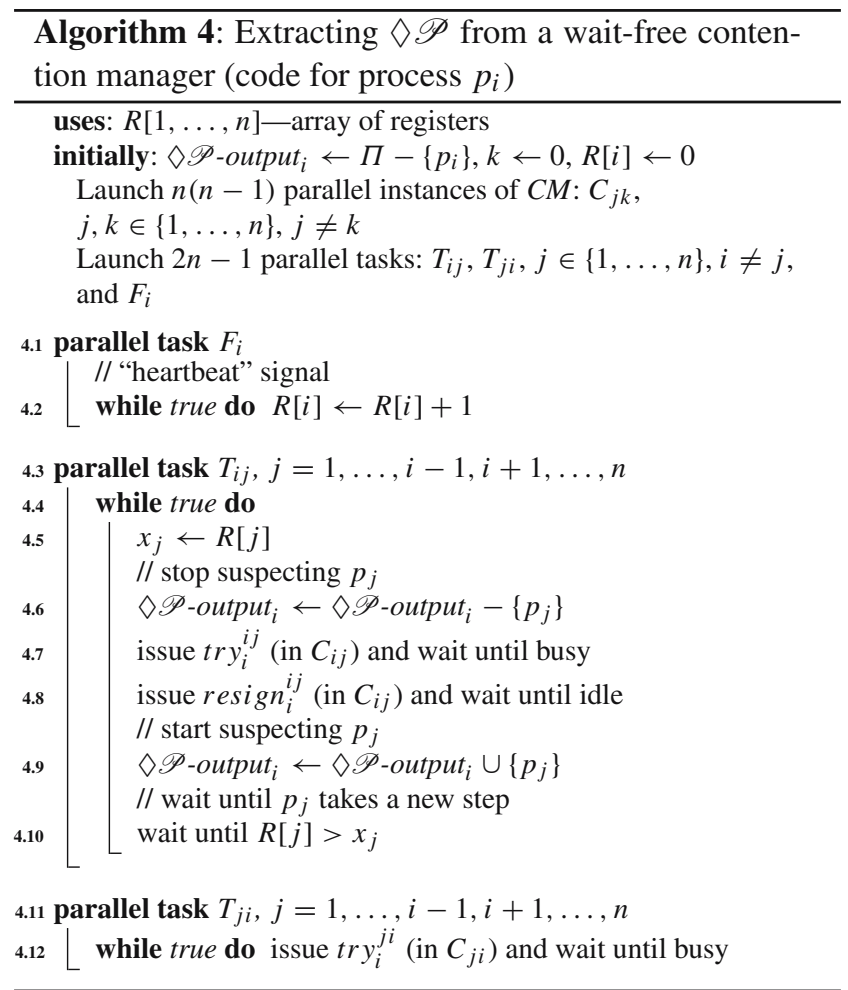

We run several instances of $C M$. These instances use disjoint sets of base shared objects and do not directly interact. Basically, in each instance, only two processes are active and all other processes are idle. One of the two processes, say $p_{j}$, gets active and never resigns thereafter, while the other, say $p_{i}$, permanently alternates between being active and idle. To $C M$ it looks like $p_{j}$ is always obstructed by $p_{i}$. Thus, to guarantee wait-freedom, the instance of $C M$ has to eventually block $p_{i}$ and let $p_{j}$ run obstruction-free until $p_{j}$ resigns or crashes. Therefore, when $p_{i}$ is blocked, $p_{i}$ can assume that 
$p_{j}$ is alive and when $p_{i}$ is busy, $p_{i}$ can suspect $p_{j}$ of having crashed, until $p_{i}$ eventually observes $p_{j}$ 's "heartbeat" signal, which $p_{j}$ periodically broadcasts using a register. This ensures the properties of $\diamond \mathscr{P}$ at process $p_{i}$, provided that $p_{j}$ never resigns.

As in Sect. 3, we face the following issue. If $p_{j}$ is correct, $p_{i}$ will be eventually blocked forever and $p_{j}$ will thus be eventually obstruction-free. Hence, in the corresponding execution, obstruction-freedom is violated, i.e., the execution cannot be produced by any OF algorithm combined with $C M$. One might argue then that $C M$ is not obliged to preserve Fairness with respect to $p_{j}$. However, we show below that, since $C M$ does not "know" how much time a process executing an OF algorithm requires to complete its operation, $C M$ has to provide $p_{j}$ with unbounded time to run in isolation.

More precisely, the processes in Algorithm 4 run $n(n-1)$ parallel instances of $C M$, denoted each $C M_{j k}$, where $j, k \in$ $\{1, \ldots, n\}, j \neq k$. We denote the events that process $p_{i}$ issues in instance $C M_{j k}$ by $t r y_{i}^{j k}$ and resign $n_{i}^{j k}$. Besides, every process $p_{i}$ runs $2 n-1$ parallel tasks: $T_{i j}, T_{j i}$, where $j \in$ $\{1, \ldots, n\}, i \neq j$, and $F_{i}$. Every task $T_{i j}$ executed by $p_{i}$ is responsible for detecting failures of process $p_{j}$. Every task $T_{j i}$ executed by $p_{i}$ is responsible for preventing $p_{j}$ from falsely suspecting $p_{i}$. In task $F_{i}, p_{i}$ periodically writes everincreasing "heartbeat" values in a shared register $R[i]$.

In every instance $C M_{i j}$, there can be only two active processes: $p_{i}$ and $p_{j}$. Process $p_{i}$ cyclically gets active (line 4.7) and resigns (line 4.8), and process $p_{j}$ gets active once and keeps getting blocked (line 4.12). Each time before $p_{i}$ gets active, $p_{i}$ removes $p_{j}$ from the list of suspected processes (line 4.6). Each time $p_{i}$ stops being blocked, $p_{i}$ starts suspecting $p_{j}$ (line 4.9) and waits until $p_{i}$ observes a "new" step of $p_{j}$ (line 4.10). Once such a step of $p_{j}$ is observed, $p_{i}$ stops suspecting $p_{j}$ and gets active again.

\section{Theorem 6 Every wait-free contention manager can be used to implement failure detector $\diamond \mathscr{P}$.}

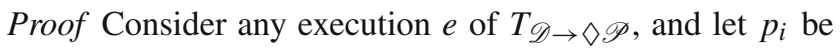
any correct process. We show that, in $e, \diamond \mathscr{P}$-output $t_{i}$ satisfies the properties of $\diamond \mathscr{P}$, i.e., $p_{i}$ eventually permanently suspects every non-correct process and stops suspecting every correct process. (Note that if a process $p_{i}$ is not correct, then $\checkmark \mathscr{P}_{\text {-output }}$ trivially satisfies the properties of $\diamond \mathscr{P}$.)

Let $p_{j}$ be any process distinct from $p_{i}$. Assume $p_{j}$ is not correct. Thus $p_{i}$ is the only correct active process in instance $C M_{i j}$. By the Fairness property of $C M, p_{i}$ is eventually obstruction-free every time $p_{i}$ becomes active, and so $p_{i}$ cannot be blocked infinitely long in line 4.7. Since there is a time after which $p_{j}$ stops taking steps, eventually $p_{i}$ starts suspecting $p_{j}$ (line 4.9) and suspends in line 4.10, waiting until $p_{j}$ takes a new step. Thus, $p_{i}$ eventually suspects $p_{j}$ forever.
Assume now that $p_{j}$ is correct. We claim that $p_{i}$ must eventually get permanently blocked so that $p_{j}$ would run obstruction-free from some point in time forever. Suppose not. Then we obtain an execution in which $p_{i}$ alternates between active and idle modes infinitely many times, and $p_{j}$ stays active and runs obstruction-free only for bounded periods of time. But the CM-history of this execution could be produced by an execution $e^{\prime}$ of some $\mathrm{OF}$ algorithm combined with $C M$ in which $p_{j}$ never completes its operation because $p_{j}$ never runs long enough in isolation. Thus, Fairness is violated in execution $e^{\prime}$ and this contradicts the assumption that $C M$ is wait-free. Hence, eventually $p_{i}$ gets permanently blocked in line 4.7. Since each time $p_{i}$ is about to get blocked, $p_{i}$ stops suspecting $p_{j}$ in line 4.6 , there is a time after which $p_{i}$ never suspects $p_{j}$.

Thus, there is a time after which, if $p_{j}$ is correct, then $p_{j}$ stops being suspected by every correct process, and if $p_{j}$ is non-correct, then every correct process permanently suspects $p_{j}$.

\subsection{The sufficiency part}

We describe an implementation of a wait-free contention manager using $\diamond \mathscr{P}$ and registers in Algorithm 5. The algorithm relies on a (wait-free) primitive GetTimestamp() that generates unique, locally increasing timestamps and makes sure that if a process gets a timestamp $t s$, then no process can get timestamps lower than $t s$ infinitely many times (this primitive can be implemented in an asynchronous system using read-write registers). The idea of the algorithm is the following. Every process $p_{i}$ that gets active receives a timestamp in line 5.2 and announces the timestamp in register $T[i]$. Every active process that invokes try repeatedly runs a leader election mechanism (lines 5.3-5.6): the non-suspected (by $\diamond \mathscr{P}$ ) process that announced the lowest (non- $\perp$ ) timestamp is elected a leader. If a process $p_{i}$ is elected, $p_{i}$ returns from $t^{\prime} y_{i}$ and becomes busy. $\diamond \mathscr{P}$ guarantees that eventually the same correct active process is elected by all active processes. All other active processes stay blocked until the process resigns and resets its timestamp in line 5.8. The leader executes steps obstruction-free then. Since the leader runs an $\mathrm{OF}$ algorithm, the leader eventually resigns and resets its timestamp in line 5.8, so that another active process, which now has the lowest timestamp in $T$, can become a leader.

Lemma 2 The contention manager implemented by Algorithm 5 guarantees wait-freedom for all OF algorithms.

Proof Consider an execution $e$ of any OF algorithm A combined with contention manager $C M$ implemented by Algorithm 5. By contradiction, assume that in $e$ some correct process is active at some time $t$ and never resigns after $t$. Let $V$ denote the non-empty set of correct processes that 


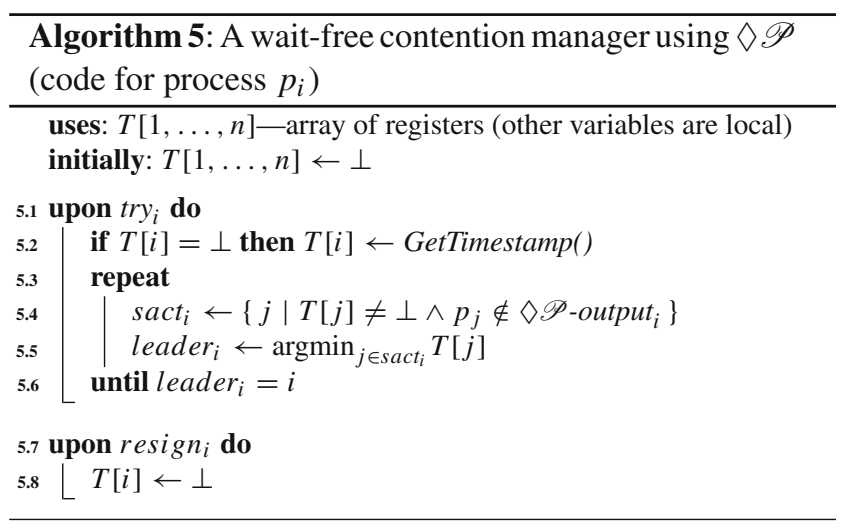

are active at some time $t$ but never resign (in line 5.8) and complete their operations thereafter, i.e., that remain active after $t$ forever. Recall that every process in $V$ either invokes try infinitely many times or invokes try and stays blocked forever (by the properties of OF algorithms). Let $t^{*}$ be time after which no process in $V$ resigns, and at which, for every process $p_{i} \in V, T[i] \neq \perp$. Let $t s_{j}^{*}$ denote the value of $T[j]$ at time $t^{*}$. Since, for every process $p_{i} \in V, t s_{i}^{*} \neq \perp$, and no process in $V$ resigns after time $t^{*}, T[i]=t s_{i}^{*}$ at all times $t \geq t^{*}$.

Let $p_{i}$ be the process in $V$ having the lowest timestamp in $\left\{t s_{k}^{*} \mid p_{k} \in V\right\}$ (there is exactly one such process since timestamps are unique). We establish a contradiction by showing that $p_{i}$ has to eventually resign.

Let us consider time $t^{\prime}>t^{*}$ after which:

- at every correct process, failure detector $\diamond \mathscr{P}$ permanently outputs the list of all non-correct processes (by the properties of $\diamond \mathscr{P}$, this eventually happens),

- all non-correct processes have crashed,

- for every correct process $p_{j} \neq p_{i}$, if $T[j] \neq \perp$, then $T[j]>t s_{i}^{*}$.

The last condition eventually holds, because timestamps are unique, no process can receive a timestamp lower that $t s_{i}^{*}$ infinitely many times and $p_{i}$ has the lowest timestamp among processes in $V$ (that retain their timestamps infinitely long).

Thus, after $t^{\prime}, p_{i}$ is always elected a leader, and every correct process $p_{j}$ other than $p_{i}$ that gets blocked after time $t^{\prime}$ will remain blocked in lines 5.3-5.6, as long as $p_{i}$ does not resign.

Hence, eventually $p_{i}$ will be the only active process that is not blocked, and thus $p_{i}$ will be given unbounded time to perform steps of $A$ in isolation. Since $A$ is obstructionfree, $p_{i}$ eventually resigns and completes its operation-a contradiction.
From Theorem 2 and Lemma 2 we immediately obtain the following result:

Theorem 7 Algorithm 5 implements a wait-free contention manager.

\section{Intermittent failure detectors}

As discussed Sect. 1, contention manager implementations based on failure detectors might be considered not very effective, especially if the goal is to reduce the complexity of executions with low contention. To cope with this issue, we revisit the notion of failure detectors and introduce here an intermittent variant of this notion.

More specifically, we introduce two intermittent failure detectors (IFDs), which can be viewed as intermittent variants of $\Omega^{*}$ and $\nabla \mathscr{P}$. We denote them by, respectively, $I_{\Omega^{*}}$ and $I_{\diamond \mathscr{P}}$. Both $I_{\Omega^{*}}$ and $I_{\diamond \mathscr{P}}$ implement two procedures that can be invoked by a contention manager: stop and query. The former stops the IFD implementation on the calling process. The latter one restarts the IFD, if it has been stopped, and queries the IFD. We assume that an IFD module at each process is, by default, stopped until the process queries the IFD for the first time.

Intuitively, $I_{\Omega^{*}}$ implements an eventual leader election mechanism among a set $S \subseteq \Pi$ of processes (given as an argument to the query procedure). When invoked by all correct processes in set $S$ sufficiently many times, with call query $(S), I_{\Omega^{*}}$ eventually permanently returns the same correct process in $S$ (a leader) at all of these processes. More precisely, $I_{\Omega^{*}}$ ensures the following property in every execution $e$. Let $S$ be a set of processes, such that every correct process in $S$ invokes query infinitely many times in $e$, and $V \subseteq S$ be the set of all correct processes in $S$. Then, $I_{\Omega^{*}}$ guarantees that in execution $e$ where (1) no process invokes stop infinitely many times and (2) all processes from set $V$ eventually permanently pass set $S$ as an argument to query: every process in $V$ eventually returns the same process $p_{l} \in V$ in every call to query $(S)$.

In the same vein, IFD $I_{\diamond \mathscr{P}}$ is similar to $\diamond \mathscr{P}$. $I_{\diamond \mathscr{P}}$ ensures the following properties. Let $V$ be a set of correct processes that, after some time, call query on $I_{\diamond} \mathscr{P}$ and never call stop thereafter, and $V^{\prime}$ be a set of (correct) processes that call query and stop on $I_{\diamond \mathscr{P}}$ infinitely many times. Call query invoked by a process $p_{i}$ returns a set of processes suspected by $p_{i} . I_{\diamond \mathscr{P}}$ guarantees that eventually: (1) every process in $V$ suspects every crashed process, and (2) no process in $V$ is ever suspected by any process in $V \cup V^{\prime}$.

We establish a formal relationship between a failure detector $\mathscr{D}$ and its intermittent variant $I_{\mathscr{D}}$ by proving that the latter provides as much information about failures as the former. We do so by treating $I_{\mathscr{D}}$ as an abstract problem and proving 
that $\mathscr{D}$ is the weakest failure detector [6] to implement $I_{\mathscr{D}}$. We say then that $\mathscr{D}$ and $I_{\mathscr{D}}$ are equivalent. In the following two theorems we establish the relationship between $I_{\Omega^{*}}$ and $\Omega^{*}$, and between $I_{\diamond \mathscr{P}}$ and $\diamond \mathscr{P}$.

Theorem $8 I_{\Omega^{*}}$ and $\Omega^{*}$ are equivalent.

Proof We show that $\Omega^{*}$ is sufficient and necessary to implement $I_{\Omega^{*}}$. The sufficiency part consists of exhibiting an algorithm that implements $I_{\Omega^{*}}$ using $\Omega^{*}$. The necessity part consists of proving that the output of $\Omega^{*}$ can be emulated using some number of instances of $I_{\Omega^{*}}$ as "black boxes" and read-write registers.

It is easy to see that one can implement $I_{\Omega^{*}}$ using $\Omega^{*}$. This can be done simply by making query $(S)$, invoked by a process $p_{i}$, return the leader elected by $\Omega^{*}$ for set $S$, and ignoring every call to stop. Therefore, $\Omega^{*}$ is sufficient to implement $I_{\Omega^{*}}$.

As $\Omega^{*}$ is a composition $\left\{\Omega_{S}\right\}_{S \subseteq \Pi, S \neq \emptyset}$, it is sufficient to prove for the necessity part that, for every non-empty subset $S$ of set $\Pi$, there is an algorithm that extracts the output of $\Omega_{S}$ from any implementation of $I_{\Omega^{*}}$.

Let $L$ be any instance of $I_{\Omega^{*}}$ and let every alive process $p_{i}$ periodically invoke query $(S)$ on $L$ and put the returned value in a local variable $\Omega_{S}$-output ${ }_{i}$. Also, assume no process ever invokes stop on $L$. Let $V$ be the set of all correct processes. Clearly, every process in $V$ will invoke query $(S)$ infinitely many times. Furthermore, every correct process in $S$ must belong to $V$. Thus, by the properties of $I_{\Omega^{*}}$, every correct process in $S$ has to eventually permanently output the id of the same correct process in $S$ in variable $\Omega_{S}$-output. Therefore, at every process $p_{i}, \Omega_{S}$-output $t_{i}$ is a valid output of failure detector $\Omega_{S}$. Hence, for every $S \subseteq \Pi, S \neq \emptyset, \Omega_{S}$ is necessary to implement $I_{\Omega^{*}}$, and so $\Omega^{*}$ is also necessary to implement $I_{\Omega^{*}}$.

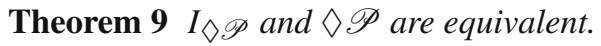

Proof We show that $\diamond \mathscr{P}$ is sufficient and necessary to implement $I \diamond \mathscr{P}$. The sufficiency part consists of exhibiting an algorithm that implements $I_{\diamond \mathscr{P}}$ using $\diamond \mathscr{P}$. The necessity part consists of showing that the output of $\diamond \mathscr{P}$ can be emulated using some number of instances of $I_{\diamond} \mathscr{P}$ as "black boxes" and read-write registers.

It is easy to see that failure detector $\diamond \mathscr{P}$ helps easily implement $I_{\diamond \mathscr{P}}$. This can be done simply by making query, invoked by a process $p_{i}$, return the set of suspected processes output by $\diamond \mathscr{P}$ at $p_{i}$, and ignoring every call to stop. Therefore, $\diamond \mathscr{P}$ is sufficient to implement $I \diamond \mathscr{P}$.

Let $D$ be any instance of $I \diamond \mathscr{P}$. Assume that every alive process $p_{i}$ periodically invokes query on $D$ and puts the

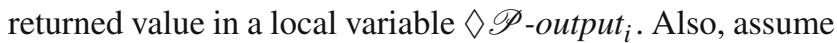
no process ever invokes stop on $D$. Let $V$ be the set of all correct processes. Clearly, every process in $V$ will invoke query infinitely many times and never invoke stop. Thus, by the properties of $I_{\diamond \mathscr{P}}$, at every process $p_{i}$ the variable $\checkmark \mathscr{P}$-output $t_{i}$ is a valid output of failure detector $\diamond \mathscr{P}$. Therefore, $\diamond \mathscr{P}$ is necessary to implement $I_{\diamond \mathscr{P}}$.

\section{Contention managers $C M_{\mathrm{nb}}$ and $C M_{\mathrm{wf}}$}

We present in this section a non-blocking contention manager $C M_{\mathrm{nb}}$ that uses IFD $I_{\Omega^{*}}$, and a wait-free contention manager $C M_{\mathrm{wf}}$ that uses IFD $I_{\diamond \mathscr{P}}$. Both contention managers stop their local IFD modules when no information about failures is needed. Moreover, when contention is low, both $C M_{\mathrm{nb}}$ and $C M_{\mathrm{wf}}$ can use any other contention manager $P C M$ that satisfies the following property:

Termination. No process is blocked infinitely long.

Therefore, a contention manager PCM that provides good average case performance (in low-contention scenarios) can be combined with the worst-case guarantees of $C M_{\mathrm{nb}}$ (nonblockingness) or $C M_{\mathrm{wf}}$ (wait-freedom).

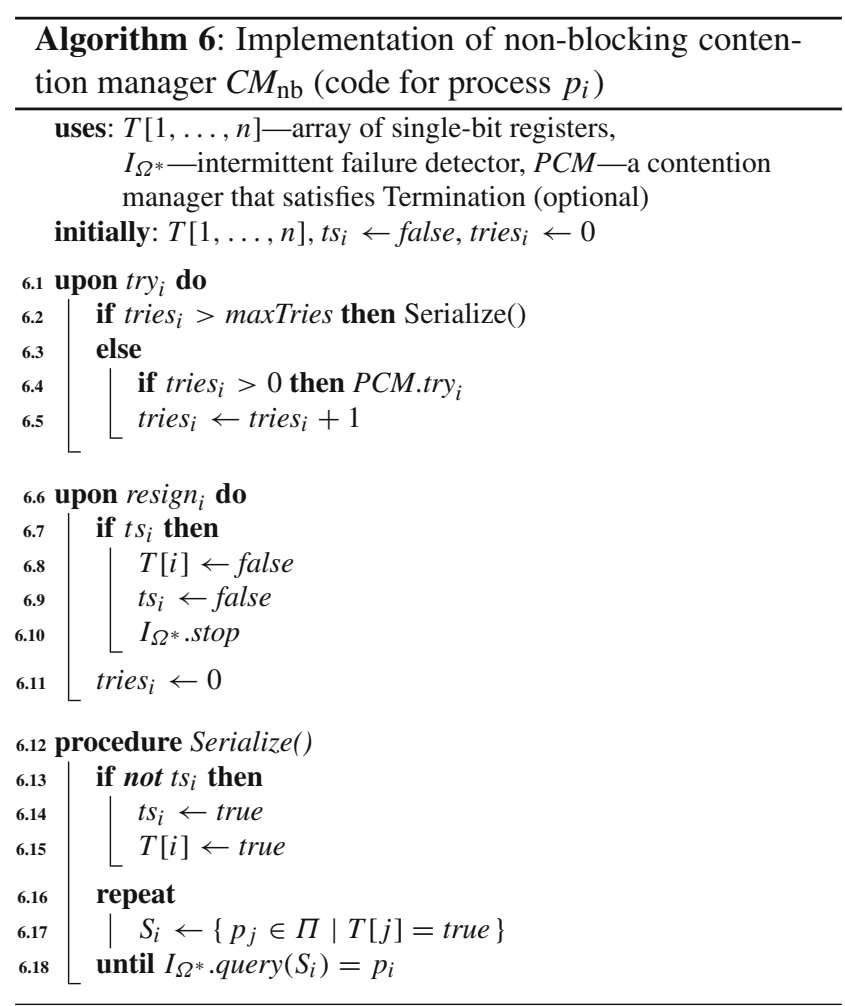

The implementation of $C M_{\mathrm{nb}}$ is shown in Algorithm 6 and the underlying idea is the following. If a process $p_{i}$ calls $t r y_{i}$ more than maxTries times before resigning, this means that $p_{i}$ cannot complete its current operation (maxTries is some 
natural constant). Thus, neither obstruction-freedom nor contention manager $P C M$ is sufficient to provide progress for $p_{i}$ anymore. In such case, $p_{i}$ enters the serialization mechanism (procedure Serialize).

The role of the serialization mechanism is to prevent livelocks. Indeed, if after some time no active process is able to complete its operation, then all active processes will eventually enter the serialization mechanism (line 6.2) and only one of them, say process $p_{i}$, will be allowed to take steps (and run obstruction-free), while others will get blocked (lines 6.166.18). Once the chosen process (leader) $p_{i}$ resigns, $p_{i}$ announces this fact to blocked processes (in line 6.8) so that they can choose another active process among them as a leader. Also when $p_{i}$ crashes, a new leader is elected.

The output of $I_{\Omega^{*}}$ is used (in line 6.18 ) only by serialized processes, i.e., by every alive process $p_{j}$ for which $T[j]=$ true. This means that module $I_{\Omega^{*}}$ can be suspended at each non-serialized process. That is why each serialized process $p_{j}$ calls stop on $I_{\Omega^{*}}$ when $p_{j}$ resigns (line 6.10). Module $I_{\Omega^{*}}$ starts working again on a process $p_{j}$ once $p_{j}$ invokes query $\left(S_{j}\right)$ again (in line 6.18).

The serialization mechanism lets only one active process take steps of an OF algorithm while blocking all others, but only when active processes eventually manage to chose a single leader among themselves in lines 6.16-6.18. If there is no agreement and so there are many leaders, none of them is guaranteed to be obstruction-free sufficiently long. If the elected leader crashes and active processes do not chose another leader, then it may happen that all active processes get blocked forever. Thus, the quality of the leader election provided by $I_{\Omega^{*}}$ is vital and we need to explain why the limited properties guaranteed by $I_{\Omega^{*}}$ are sufficient.

Intuitively, $C M_{\mathrm{nb}}$ guarantees non-blockingness when the leader election provided by $I_{\Omega^{*}}$ is eventually accurate. However, $I_{\Omega^{*}}$, as used by $C M_{\mathrm{nb}}$, guarantees the accuracy of the leader election only in executions in which non-blockingness is violated. Thus, if there existed an execution of an $\mathrm{OF}$ algorithm combined with $C M_{\mathrm{nb}}$ in which non-blockingness did not hold, $I_{\Omega^{*}}$ would have to guarantee eventually accurate leader election in this execution, in which case $C M_{\mathrm{nb}}$ would have to guarantee non-blockingness. Hence, such an execution is effectively impossible.

More precisely, if in an execution $e$ non-blockingness is violated, this means that at some point in time $t$ there are some correct active processes (a set $V$ ) and no process resigns thereafter. But then all these processes will keep querying $I_{\Omega^{*}}$ forever, eventually permanently about the same set of processes $S$. Furthermore, no process ever stops $I_{\Omega^{*}}$ after time $t$, for $I_{\Omega^{*}}$ may be stopped only by a process that resigns. Thus, eventually $I_{\Omega^{*}}$ will make processes in set $V$ output a single correct active process as their leader from some point in time forever. The elected leader will then be eventually obstruction-free, in which case the leader has to eventually complete the operation it executes and resign — contradicting our assumption that no process resigns after time $t$.

Lemma 3 Contention manager $C M_{\mathrm{nb}}$ shown in Algorithm 6 guarantees non-blockingness for every OF algorithm.

Proof By contradiction, assume that in some execution $e$ of an $\mathrm{OF}$ algorithm $A$ combined with contention manager $C M_{\mathrm{nb}}$ non-blockingness is violated. This means that there exists time $t$, such that some correct processes are active at $t$ and no process resigns after $t$. Let us denote by $t^{\prime}$ a point in time after $t$, such that (1) only correct processes are alive after $t^{\prime}$ and (2) no process that is idle at $t^{\prime}$ becomes active after $t^{\prime}$. Let us denote by $V$ the set of processes that are active at $t^{\prime}$. Clearly, each process in $V$ is permanently active from time $t^{\prime}$ forever.

For each correct process $p_{i} \notin V$ the value $T[i]$ is eventually permanently set to false after $t^{\prime}$, for $p_{i}$ had to resign before $t$ or $p_{i}$ is never active in $e$, and $p_{i}$ can set $T$ [i] to true (in line 6.15) only when $p_{i}$ is active. Each faulty process must have crashed by $t^{\prime}$. Therefore, there exists a time after which, for each process $p_{i} \notin V$, the value of $T[i]$ will not change.

Each process $p_{i}$ in set $V$ has to periodically invoke $t r y_{i}$, until $p_{i}$ gets blocked forever (for execution $e$ has to be wellformed). However, $p_{i}$ can get blocked forever only in procedure Serialize, for PCM satisfies Termination. Thus, after $t$, each process in $V$ will eventually enter procedure Serialize in line 6.2, because after $t$ the value of tries $_{i}$ cannot be reset to 0 in line 6.11, as no process resigns after $t$, and tries $_{i}$ increases in line 6.5 each time $p_{i}$ calls $t r y_{i}$ and does not enter procedure Serialize. Thus, if at time $t$ the value of $T[i]$ is false, $p_{i}$ will eventually set the value to true. Furthermore, $T[i]$ cannot be reset to false after $t$ as $p_{i}$ does not resign after $t$. Therefore, there is a time $t^{\prime \prime}>t^{\prime}$, such that after $t^{\prime \prime}$ : (1) for every process $p_{i} \in V$ the value $T[i]$ will be permanently set to true, and (2) for every process $p_{j} \notin V$ the value $T[j]$ will not change.

Denote by $S$ the set of processes for which $T[\ldots]=$ true after time $t^{\prime \prime}$. Clearly, $V \subseteq S$ because for every process $p_{i} \in V$ the value of $T[i]$ is permanently set to true after $t^{\prime \prime}$. Also, no process invokes stop infinitely many times (in line 6.10) for no process resigns infinitely often in $e$. Furthermore, after time $t^{\prime \prime}$ only processes in set $V$ will be querying $I_{\Omega^{*}}$ in line 6.18 , and eventually all processes in $V$ will be querying $I_{\Omega^{*}}$ about set $S$, constructed in line 6.17, which never changes after $t^{\prime \prime}$. Therefore, eventually, at each process $p_{i}$ in set $V$, the module $I_{\Omega^{*}}$ will be permanently returning the same process $p_{l} \in V$ in each call to query $\left(S_{i}=S\right)$ in line 6.18. Thus, eventually only process $p_{l}$ will always return from procedure Serialize.

Therefore, eventually all processes in set $V$, except for $p_{l}$, will be blocked in lines $6.16-6.18$ forever and process $p_{l}$ will execute infinitely many steps of algorithm $A$ 
obstruction-free. But then $p_{l}$, by obstruction-freedom of $A$, has to eventually resign and complete its current operation of $A$. Thus, $p_{l} \notin V-$ a contradiction.

From Theorem 1 and Lemma 3 we immediately obtain the following result:

Theorem 10 Contention manager $C M_{\mathrm{nb}}$ is non-blocking.

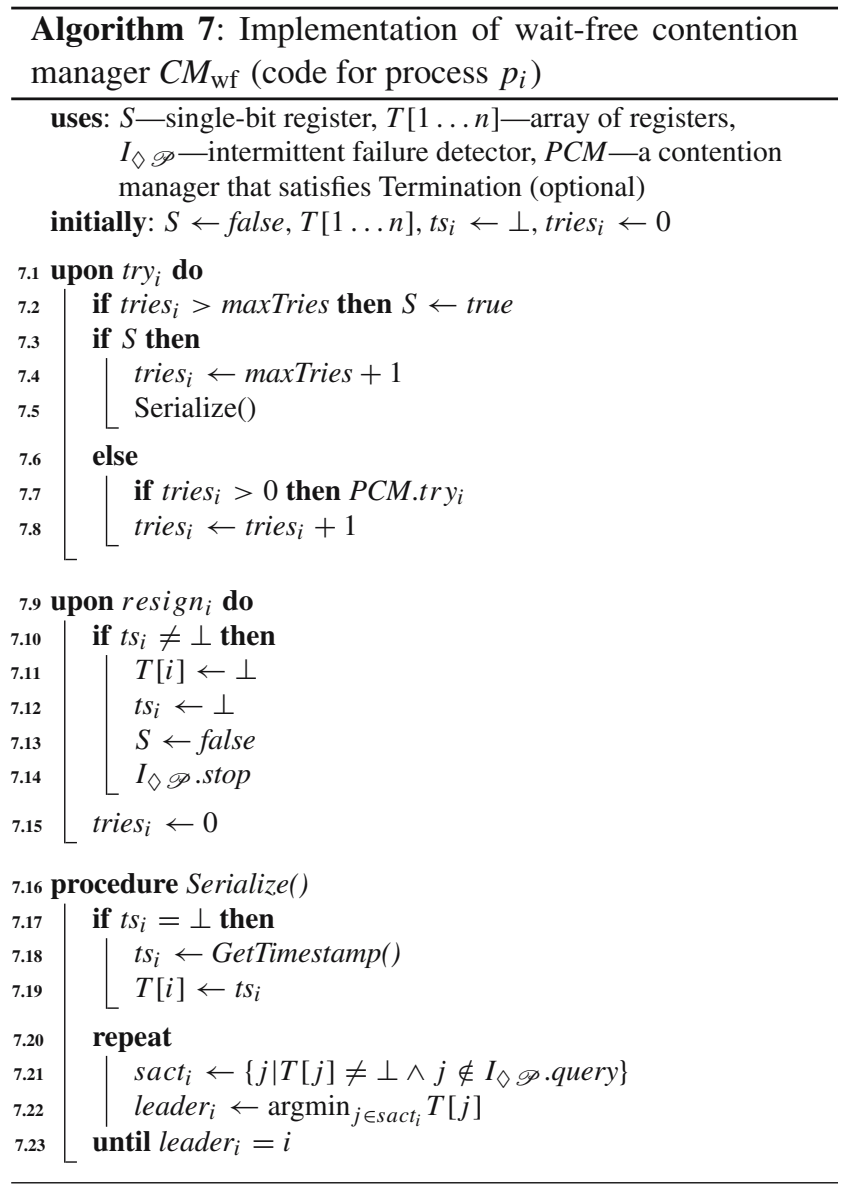

The implementation of $C M_{\mathrm{wf}}$ is presented in Algorithm 7. The algorithm relies on a (wait-free) function GetTimestamp() for generating unique timestamps such that if some process gets a timestamp $t s$ then no process gets a timestamp lower than $t s$ infinitely many times. Such a timestamping mechanism can be easily implemented with registers.

The basic idea of $C M_{\mathrm{wf}}$ is the following. When an active process $p_{i}$ invokes $t r y_{i}$ more than maxTries times, $C M_{\mathrm{wf}}$ sets flag $S$ to true in line 7.2 and starts serializing all reported operations. As long as flag $S$ is raised, every new process that invokes try enters immediately the serialization mechanism (procedure Serialize).

The serialization mechanism works as follows. First, $p_{i}$ gets a timestamp in line 7.18 and announces the timestamp in array $T$ in line 7.19 . Then, using $I_{\diamond} \mathscr{P}, p_{i}$ periodically runs a leader election mechanism: the non-suspected process that announced the lowest timestamp in $T$ is elected a leader. If $p_{i}$ is a leader, $p_{i}$ returns from the serialization mechanism.

$I_{\diamond \mathscr{P}}$ guarantees that eventually the same correct active process is elected leader by all serialized processes (unless these processes resign before). The leader executes steps of the OF algorithm obstruction-free and so it eventually resigns. After doing so, the leader resets its timestamp in lines 7.11 and 7.12 so that the active process that currently has the lowest timestamp can become a leader thereafter. When a serialized process finishes its operation, it sets flag $S$ to false in line 7.13. As a result, once all concurrent serialized operations are completed, the processes might fall back to some other, may be more pragmatic, contention management scheme (provided by contention manager $P C M$ ).

It might not be straightforward to see why the properties of $I_{\diamond \mathscr{P}}$ are strong enough for the serialization mechanism. Similarly to $I_{\Omega^{*}}$, IFD $I_{\diamond \mathscr{P}}$, when used with contention manager $C M_{\mathrm{wf}}$, provides useful information only in executions in which wait-freedom is violated. Consider then an execution $e$ of an $\mathrm{OF}$ algorithm combined with $C M_{\mathrm{wf}}$. If in $e$ wait-freedom is violated, there are some correct processes (a set $V$ ) that are active from some point in time $t$ forever. These processes will at some time query $I_{\diamond \mathscr{P}}$ and never stop $I_{\diamond \mathscr{P}}$ thereafter. But then, by properties of $I_{\diamond \mathscr{P}}$, processes in set $V$ will be eventually never suspected by any other active process. Thus, all the active processes have to eventually elect the correct process with the lowest timestamp (in $V$ ) as their leader and let the process run obstruction-free forever. But the leader will have to eventually complete its operation then, and so it will not be active forever-contradicting our assumption.

Lemma 4 Contention manager $C M_{\mathrm{wf}}$ implemented by Algorithm 7 guarantees wait-freedom for all OF algorithms.

Proof By contradiction, assume that in some execution $e$ of some $\mathrm{OF}$ algorithm $A$ combined with contention manager $C M_{\mathrm{wf}}$ there are some correct processes (a set $V$ ) that do not complete their operations, i.e., from some point in time they are active forever. By properties of OF algorithms, each process from set $V$ has to invoke try infinitely many times, unless the process gets blocked forever. However, the latter can happen only after the process gets serialized (i.e., enters procedure Serialize) and after the process receives and announces its timestamp in line 7.18 and line 7.19 , respectively. That is because contention manager PCM satisfies Termination and so $P C M$ cannot block any process forever in line 7.7.

Claim For every process $p_{j}$ in set $V$ there is a timestamp $t s_{j}^{F} \neq \perp$ such that eventually $t s_{j}=t s_{j}^{F}$ forever.

Proof Let us take some process $p_{j}$ in set $V$ and denote by $t$ a point in time after which $p_{j}$ is active forever. Clearly, after 
$t$ process $p_{j}$ cannot reset its timestamp to $\perp$ (in line 7.12) because $p_{j}$ does not resign after $t$. Thus, by the condition in line 7.17, once $p_{j}$ receives a timestamp after time $t, p_{j}$ will retain this timestamp forever.

Assume then, by contradiction, that $p_{j}$ has its timestamp equal to $\perp$ from time $t$ forever. But after $t$ process $p_{j}$ is permanently active and thus $p_{j}$ eventually has to enter the serialization mechanism, after calling $t^{\prime} y_{j}$ at most maxTries +1 times. But then $p_{j}$ will receive a non- $\perp$ timestamp in line 7.18 after $t$-a contradiction.

Let us denote by $p_{i}$ the process having the lowest timestamp in $\left\{t s_{k}^{F} \mid p_{k} \in V\right\}$ (there is always one, and only one, such a process, by the claim proved before and because timestamps are unique). We will lead to a contradiction by showing that $p_{i}$ has to eventually complete its current operation and resign.

Firstly, let us observe that all processes in set $V$ will query $I_{\diamond \mathscr{P}}$ in line 7.21 infinitely many times and after some time they will never invoke stop anymore in line 7.14. Therefore, by properties of $I_{\diamond \mathscr{P}}$, eventually every process $p_{j} \in$ $V$ will permanently suspect every crashed process and will never suspect any other process from set $V$ anymore. Therefore, eventually all processes in set $V$, except for $p_{i}$, will be blocked forever in lines 7.20-7.23, because $p_{i}$ is in set $V$ and $p_{i}$ has the lowest timestamp from all processes in set $V$.

Let us consider time $t$ after which:

- the failure detection at processes in set $V$ (provided by $I_{\diamond \mathscr{P})}$ is already accurate,

- only correct processes are alive,

- $p_{i}$ has already got its timestamp $t s_{i}=t s_{i}^{F}$ in line 7.18 and announced it in line 7.19, and

- all active processes other than $p_{i}$ have timestamps larger than $t s_{i}$ or equal to $\perp$.

The last condition will surely eventually hold in execution $e$ because of the following reasons. First, timestamps are unique. Second, no process can get a timestamp lower than $t s_{i}^{F}$ infinitely many times. Third, $p_{i}$ has the lowest timestamp from all correct processes that never become idle after some point in time (set $V$ ) and so keep their once received timestamp forever.

Clearly, process $p_{i}$ cannot be blocked infinitely long. Furthermore, all processes from set $V$, except for $p_{i}$, will eventually be blocked forever. This means that the only processes that can obstruct $p_{i}$ infinitely many times (i.e., that can execute infinitely many steps of OF algorithm $A$ concurrently with $p_{i}$ ) are these processes that complete infinitely many operations and thus call try and resign infinitely many times. Let us denote the set of these processes by $V^{\prime}$. If we prove that $V^{\prime}$ is empty, then we show that from some point in time process $p_{i}$ is obstruction-free forever and so, by obstruction-freedom, has to eventually complete its current operation and resign - a contradiction with our assumption that $p_{i} \in V$.

Claim Set $V^{\prime}$ is empty.

Proof Suppose not-that there are some processes that belong to $V^{\prime}$, i.e., processes that invoke try and resign infinitely many times. Process $p_{i}$ sets flag $S$ to true in line 7.2 infinitely many times, because $p_{i}$ must execute line 7.4 after time $t$ and cannot reset tries $_{i}$ thereafter. Therefore, there has to be some process $p_{j} \in V^{\prime}$ that observes $S=$ true in line 7.3 and enters procedure Serialize in line 7.5 infinitely many times. This is because flag $S$ can be reset to false only by a process that observes $S=$ true (and thus enters the serialization mechanism) and resigns, and $S$ is set to true infinitely many times by $p_{i}$. Process $p_{j}$ will then invoke query and stop on $I_{\diamond \mathscr{P}}$ infinitely often. But $p_{j}$ will always have a timestamp larger than $t s_{i}^{F}$ after time $t$ and, by properties of $I_{\diamond \mathscr{P}}$, $p_{j}$ will eventually never suspect process $p_{i} \in V$. Thus, eventually process $p_{j}$ will be blocked forever and so $p_{j} \notin V^{\prime}$-a contradiction.

From Theorem 2 and Lemma 4 we immediately obtain the following result:

Theorem 11 Contention manager $C M_{\mathrm{wf}}$ is wait-free.

\section{Implementation of IFDs $I_{\Omega^{*}}$ and $I_{\diamond \mathscr{P}}$}

Precisely because $I_{\Omega^{*}}$ and $I_{\diamond \mathscr{P}}$ are sufficient to implement a non-blocking contention manager, they are impossible to implement in an asynchronous system. It is however usually reasonable to assume eventual synchrony, which means that eventually there exists an upper and a lower bound on the time it can take for a process to execute a step. These bounds are not known to processes, can be arbitrary and also can be different in each execution.

An example implementation of $I_{\diamond \mathscr{P}}$ in an eventually synchronous system, similar to known message passing implementations of $\diamond \mathscr{P}[1,7,9,25]$, is presented in Algorithm 8 . The idea of the algorithm is the following. Each process $p_{i}$, for which IFD is not stopped, periodically increments a "heartbeat" register $A[i]$. Process $p_{i}$ also checks the registers $A[\ldots]$ of other processes. If the value in a register $A[j]$ of process $p_{j}$ has not changed since the last read, then $p_{i}$ starts suspecting $p_{j}$ (which means that a correct processes that never queries $I_{\diamond \mathscr{P}}$ can be eventually permanently suspected). If $p_{i}$ observes later that $p_{j}$ has incremented its register, then $p_{i}$ stops suspecting $p_{j}$ and increases its timeout value. This timeout tells $p_{i}$ how many steps $p_{i}$ has to perform between two checks of the registers of other processes. Eventually $p_{i}$ adjusts its timeout to the slowest process, provided that $p_{i}$ is running $I_{\diamond \mathscr{P}}$ for sufficiently long time. 


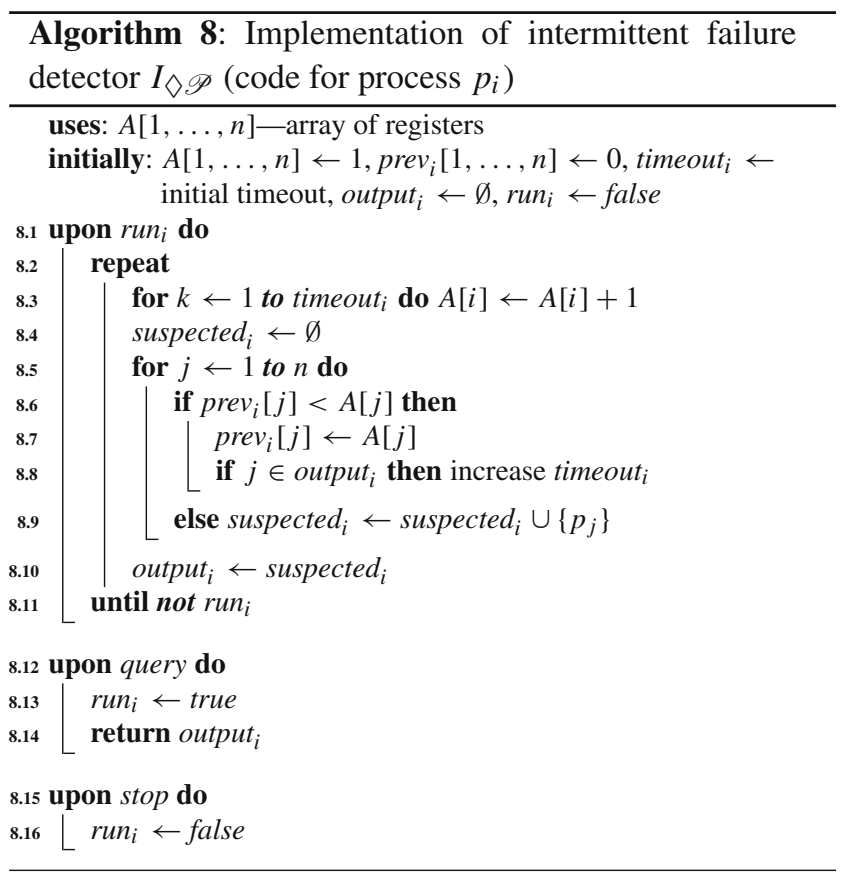

\section{Theorem 12 Algorithm 8 implements $I_{\diamond} \mathscr{P}$.}

Proof Let us denote by $V$ the set of correct processes that at some point in time call query and never call stop thereafter. Let us denote by $V^{\prime}$ the set of processes that call query and stop infinitely many times. Let us take a point in time $t$, such that after $t$ every process $p_{j} \in V$ has its value of $r u n_{j}$ equal to true forever.

If a process $p_{i}$ crashes, then $p_{i}$ will no longer increment the value in $A[i]$ in line 8.3. As run ${ }_{j}=$ true at every process $p_{j} \in V$ after $t$, all processes in $V$ will eventually execute the "repeat" loop in lines 8.2-8.11 twice after the crash of $p_{i}$, and observe that $A[i]$ has not changed (in line 8.6). Thus, every process $p_{j} \in V$ will eventually add $p_{i}$ to its set suspected ${ }_{j}$ in line 8.9. Therefore, eventually $p_{i}$ will be suspected by all processes in $V$ and thus we have proved property 1 of $I_{\diamond \mathscr{P}}$.

Now let us prove property 2. Assume, by contradiction, that a process $p_{i} \in V$ is suspected infinitely often by a process $p_{j} \in V \cup V^{\prime}$. Process $p_{i}$ is in $V$ and so, after time $t, \operatorname{run}_{i}=$ true forever. Therefore, $p_{i}$ will increment its register $A[i]$ infinitely many times in line 8.3. Process $p_{j}$ is in $V \cup V^{\prime}$ and so the condition run $_{j}=$ true is satisfied infinitely many times, which means that $p_{j}$ will execute the loop in lines 8.2-8.11 infinitely often. Therefore, $p_{j}$ will observe in line 8.6 infinitely many times that $A[i]$ has changed and so, as $p_{j}$ suspects $p_{i}$ infinitely often, $p_{j}$ will increase its timeout in line 8.8 infinitely many times. It means that at some point in time timeout $j$ will be so large that $p_{j}$ will spend much more time in the loop in line 8.3 (consisting of timeout ${ }_{j}$ steps) than it will take $p_{i}$ to execute the code in lines $8.4-8.10$ and increment $A[i]$ at least once in line $8.3(2 n+1$ steps, which is constant in any given execution). This is because eventually there exists an upper and a lower bound on the time it can take for any process to take a step, and thus also the relative speed of the processes $p_{i}$ and $p_{j}$ is eventually bounded. Therefore, between any two checks of $p_{j}, p_{i}$ will manage to increment $A[i]$, and so $p_{i}$ will not be ever suspected by $p_{j}-$ a contradiction.

IFD $I_{\Omega} *$ can be implemented in a similar way. In fact, one can easily extract the output of $I_{\Omega^{*}}$ using $I_{\diamond \mathscr{P}}$ : query $(S)$ invoked on $I_{\Omega^{*}}$ would then return this alive (i.e., non-suspected by $I \diamond \mathscr{P}$ ) process in set $S$ that has the lowest identifier. Clearly, $I_{\Omega} *$ can be implemented in a more efficient way if $I_{\diamond \mathscr{P}}$ is not used, for we can make only the elected leader send "heartbeat" signals to others, unlike in the presented implementation of $I \diamond \mathscr{P}$ in which every alive process for which IFD is not stopped has to keep incrementing its "heartbeat" counter.

A more effective implementation of $I \diamond \mathscr{P}$, assuming an eventually synchronous system, is presented in Algorithm 9. The idea is straightforward: an alive process $p_{i}$ with the lowest identifier among the processes that participate in the leader election is elected (line 9.4). Then $p_{i}$ permanently increments its register $A[i]$ to inform others that $p_{i}$ is still alive (line 9.6). If a process $p_{j}$ observes that $A[i]$ has not changed since the last read, $p_{j}$ suspects $p_{i}$ of having crashed and elects a new leader. If later $p_{j}$ discovers that $p_{i}$ is alive, $p_{j}$ increases the timeout $_{j}$ value (line 9.8) which tells $p_{j}$ how long $p_{j}$ should wait (in line 9.9) between any two checks of a register $A[i]$.

\section{Theorem 13 Algorithm 9 implements $I_{\Omega} *$.}

Proof Assume, by contradiction, that Algorithm 9 does not implement $I_{\Omega^{*}}$. This means that there exists some execution $e$ in which the property of $I_{\Omega} *$ is violated.

Denote by $V$ the set of processes that invoke query infinitely many times in $e$. Assume that there exists a set $S$ of processes such that: (1) all correct processes in $S$ belong to $V$, and (2) starting from some time $t$, all processes in set $V$ periodically invoke query $(S)$. Assume also that no process invokes stop infinitely many times in $e$. Let us denote by $p_{l}$ the process from set $V$ that has the lowest identifier. We will lead to a contradiction by showing that all processes in $V$ have to eventually permanently return $p_{l}$ in every call to query $(S)$.

All correct processes from set $S$ are in $V$, and $p_{l}$ has the lowest identifier from all processes in $V$. Therefore, every process from set $S$ that has the identifier lower than the identifier of $p_{l}$ eventually crashes in $e$. Therefore, eventually no process $p_{i} \in S$ such that $i<l$ ( $i$ and $l$ are the identifiers of process $p_{i}$ and $p_{l}$, respectively) will increment its register 


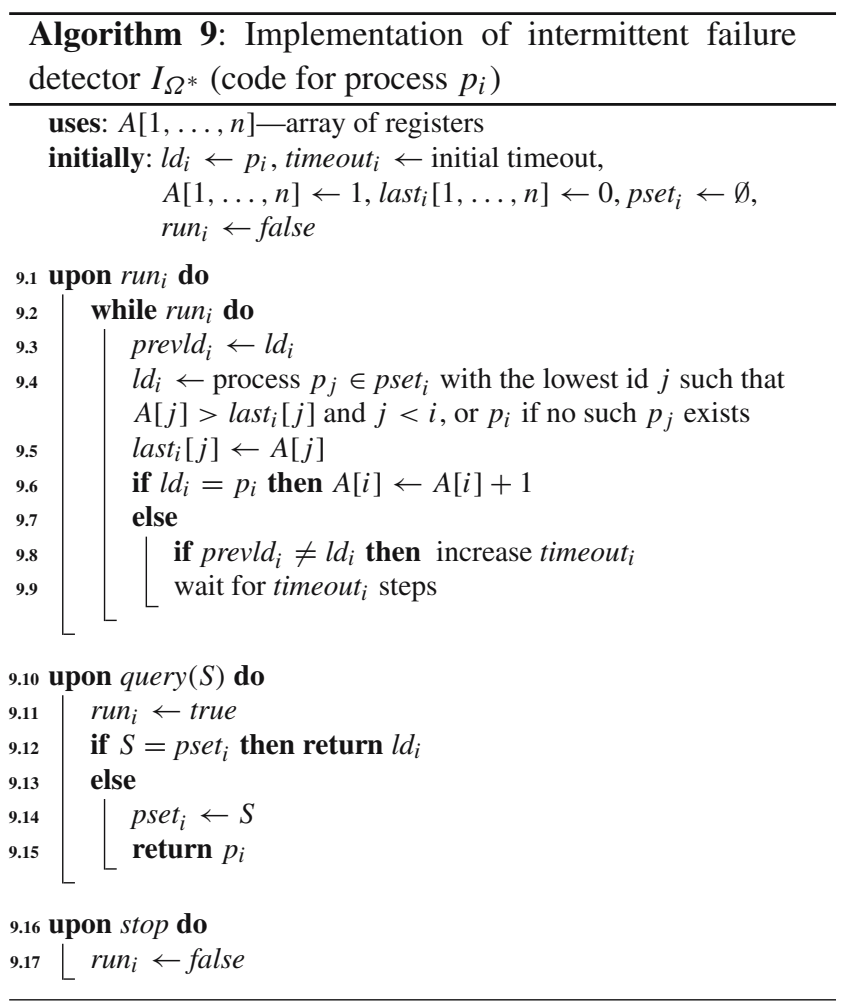

$A[i]$. This means that process $p_{l}$ will eventually permanently elect itself a leader in line 9.4, because $p_{l}$ has the lowest id from all correct processes in pset $_{l}$ and eventually pset $t_{l}$ is permanently equal to $S$ (as $p_{l} \in V$ ). Therefore, eventually process $p_{l}$, after some time $t^{\prime}$, will be periodically incrementing its register $A[l]$ in line 9.6 and never wait in line 9.9 anymore.

Suppose some process $p_{i} \in V, i \neq l$, never permanently elects $p_{l}$ as its leader. As $p_{l}$ is permanently increasing its register $A[l]$ after time $t^{\prime}$, process $p_{i}$ has to observe infinitely many times in line 9.4 that $A[l]$ has changed, elect $p_{l}$ a leader and wait for timeout $t_{i}$ steps in line 9.9. Process $p_{i}$ also infinitely many times elects other process as its leader, and so $p_{i}$ has to increment the value of timeout $_{i}$ infinitely many times in line 9.8 .

Process $p_{l}$, after time $t^{\prime}$, executes a constant (for a given number of processes) number of steps between two increments of $A[l]$ in line 9.6. As the system is eventually synchronous, there is an upper bound $t_{\max }$ on the time between any two increments of $A[l]$ by process $p_{l}$. There is also (eventually) a lower bound, $t_{\min }$, on the time in which process $p_{i}$ can execute a single step in line 9.9. Therefore, there exists such a value of timeout $_{i}$ that $t_{\min } \cdot$ timeout $_{i}>t_{\max }$. Thus, eventually process $p_{i}$ will have to wait in line 9.9 longer than it may take for $p_{l}$ to increment $A[l]$. This means that eventually $p_{i}$ will observe that $A[l]>$ last $_{i}[l]$ in every execution of line 9.4 and so $p_{i}$ will eventually permanently elect $p_{l}$ as a leader-a contradiction.
Algorithms 8 and 9 use an array $A$ of $n$ unbounded registers for simplicity. In fact, $A$ can be replaced by an array of $2 n^{2}$ single-bit registers $\operatorname{send}_{i j}, i, j=1, \ldots, n$. Instead of incrementing $A[i]$, a process $p_{i}$ would set send $d_{i j}$ to true for all $j=1, \ldots, n$, and instead of comparing $A[i]$ to $\operatorname{prev}_{j}[i]$, process $p_{j}$ would check whether send $d_{i j}$ is true and reset send $_{i j}$ to false. Such an optimized implementation of $I_{\diamond \mathscr{P}}$ or $I_{\Omega^{*}}$ uses $O\left(n^{2}\right)$ memory.

\section{Overhead of contention management}

We discuss now the inherent overhead of contention management. In general, a process that executes operations implemented by an obstruction-free object implementation without any contention should call try only few timesideally, once per operation. In fact, in executions without contention, obstruction-freedom is strong enough a liveness guarantee and a contention manager is not needed. Thus, it seems desirable to minimize the number of steps of a contention manager that a process executes within any operation, during which try has been invoked only once, at the beginning of the operation.

It is easy to see that when try is invoked for the first time within a given operation (if maxTries $>0$ ), contention manager $C M_{\mathrm{nb}}$ makes no process access the intermittent failure detector or a shared object. On the contrary, a process calling try implemented by contention manager $C M_{\mathrm{wf}}$ always performs at least one step, accessing shared register $S$. Thus, $C M_{\mathrm{nb}}$ guarantees that no process will execute a step of the contention manager (in a call try or resign) in an operation, during which try is called only once. Contention manager $C M_{\mathrm{wf}}$, however, will always make processes access shared objects (execute steps) inside every call try, even in executions with no contention. The following theorem states that this is inherent to any wait-free contention managers.

Theorem 14 Let $A$ be any OF algorithm. There is no waitfree contention manager $C M$ that guarantees the following: For every execution e of A combined with CM, every process $p_{i}$, and every operation invoked at a time $t$ and completed at a time $t^{\prime}$ in e by process $p_{i}$, if there is only one event tryinv in period $\left(t, t^{\prime}\right)$ in e|i, then there is no step of CM in period $\left(t, t^{\prime}\right)$ in e $\mid i$.

Proof Assume, by contradiction, that such a contention manager $C M$ exists. Consider two correct processes: $p_{1}$ and $p_{2}$, executing an $\mathrm{OF}$ algorithm $A$ presented in Algorithm 1 (in Sect. 2.3). It is easy to verify that $A$ is an OF algorithm.

To establish a contradiction, we construct an execution of $A$ combined with $C M$ in which correct process $p_{1}$ can never complete its operation, i.e., wait-freedom is violated. Let $p_{1}$ and $p_{2}$ be correct processes and consider the following execution $e$ : 
1. Process $p_{1}$ starts executing operation of-getTimestamp and reaches line 1.5 .

2. Process $p_{1}$ executes, then, steps in lines 1.5-1.6 for some value of $j$ and suspends its execution for some time.

3. Then process $p_{2}$ starts executing operation of-getTimestamp, completes the operation and resigns. Clearly, $p_{2}$, while executing the operation, is obstruction-free and thus eventually has to complete the operation.

4. Next, $p_{1}$ continues executing steps and observes in line 1.8 that $A[j]=2$. Thus, $p_{1}$ is not able to complete the operation and has to start the next iteration of the "while" loop.

5. Steps 2-5 are repeated forever.

To see why steps $2-5$ can be repeated infinitely many times in execution $e$, notice that process $p_{2}$ invokes $t r y_{2}$ only once per each operation $p_{2}$ executes in $e$. That is, $p_{2}$ invokes $t r y_{2}$ only when $p_{2}$ is idle. This means that, by our assumptions about $C M, p_{2}$ does not execute any step on behalf of $C M$ in execution $e$. Therefore, $H_{\mathrm{OF}}(e)|2=e| 2$, and so for the module of contention manager $C M$ executed at process $p_{2}$ execution $e$ is indistinguishable from an execution $e^{\prime}=e \mid 2$ (in which only process $p_{2}$ is ever active). Thus, there is such an execution $e^{\prime}=e \mid 2$, in which process $p_{2}$ is never delayed by $C M$ for sufficiently long time, so that $p_{1}$ could complete its operation and resign. Therefore, execution $e$ of an OF algorithm combined with $C M$, in which wait-freedom is violated, exists - a contradiction with the assumption that $C M$ is wait-free.

\section{Concluding remarks}

It is often argued that contention is rare in many practical settings. Therefore, it is very appealing to design algorithms that are optimized for the case when processes do not obstruct each other. Ensuring progress in the worst-case scenarios is still important, though, but can be delegated to specialized contention management oracles that, once devised, may be reused for various object implementations. This approach reduces the programmer's problem of designing a wait-free (resp. non-blocking) algorithm to guaranteeing obstructionfreedom, which is commonly perceived as being easier.

In this paper, we determined the minimal failure information to implement contention managers that provide strong progress guarantees (non-blockingness or wait-freedom) for all obstruction-free algorithms. Namely, we proved that failure detectors $\Omega^{*}$ and $\diamond \mathscr{P}$ are the weakest to implement any non-blocking and wait-free contention manager, respectively. The proofs include concrete contention manager implementations that use these failure detectors. These implementations are interesting in their own right.
We argued, however, that there is a drawback in building contention managers that use failure detectors. Namely, the very notion of a failure detector induces a systematic cost of detecting process crashes, even when no information about failures is needed, e.g., in executions with low contention, in which obstruction-freedom is strong enough to guarantee progress. Our solution to this problem is the abstraction of an intermittent failure detector, which is of independent interest. This abstraction encapsulates a failure detection mechanism that knows of, and responds to, contention manager demands, yet can still be described with axiomatic properties and compared precisely to a failure detector. We showed that two intermittent failure detectors described in this paper, which are equivalent to failure detectors $\Omega^{*}$ and $\diamond \mathscr{P}$, can be indeed used in an implementation of a, respectively, non-blocking and wait-free contention manager.

The contention managers we present in this paper do not, by default, exhibit good average-case performance. In most cases, they would simply serialize all concurrent operations, including those that concern disjoint sets of memory locations. This does not exploit situations when such operations can be safely run in parallel. Fortunately, as we show through Algorithm 6 and 7, our contention managers can be easily composed with contention management strategies that perform well in the average case but do not ensure any worst-case guarantees (namely non-blockingness or wait-freedom) [27, $28]$.

Acknowledgments We are very grateful to Hagit Attiya, Maurice Herlihy, Bastian Pochon, Faith Fich, Victor Luchangco, Mark Moir and Nir Shavit for interesting discussions on the topic of this paper. We would also like to thank the anonymous reviewers for helpful comments.

\section{References}

1. Aguilera, M.K., Delporte-Gallet, C., Fauconnier, H., Toueg, S.: Stable leader election. In: Proceedings of the International Symposium on Distributed Computing (DISC) (2001)

2. Attiya, H., Guerraoui, R., Kouznetsov, P.: Computing with reads and writes in the absence of step contention. In: Proceedings of the 19th International Symposium on Distributed Computing (DISC) (2005)

3. Attiya, H., Welch, J.L.: Distributed Computing: Fundamentals, Simulations and Advanced Topics, 2nd edn. Wiley, New York (2004)

4. Bershad, B.N.: Practical considerations for non-blocking concurrent objects. In: Proceedings of the 14th IEEE International Conference on Distributed Computing Systems (ICDCS), pp. 264-273 (1993)

5. Boichat, R., Dutta, P., Frølund, S., Guerraoui, R.: Deconstructing Paxos. ACM SIGACT News Distributed Computing Column 34(1), 47-67 (2003). Revised version of EPFL Technical Report 200106, January 2001

6. Chandra, T.D., Hadzilacos, V., Toueg, S.: The weakest failure detector for solving consensus. J. ACM 43(4), 685-722 (1996) 
7. Chandra, T.D., Toueg, S.: Unreliable failure detectors for reliable distributed systems. J. ACM 43(2), 225-267 (1996)

8. Dwork, C., Lynch, N.A., Stockmeyer, L.J.: Consensus in the presence of partial synchrony. J. ACM 35(2), 288-323 (1988)

9. Fetzer, C., Raynal, M., Tronel, F.: An adaptive failure detection protocol. In: Proceedings of the 2001 Pacific Rim International Symposium on Dependable Computing (2001)

10. Fich, F., Luchangco, V., Moir, M., Shavit, N.: Obstruction-free algorithms can be practically wait-free. In: Proceedings of the 19th International Symposium on Distributed Computing (DISC) (2005)

11. Fischer, M.J., Lynch, N.A., Paterson, M.S.: Impossibility of distributed consensus with one faulty process. J. ACM 32(3), 374382 (1985)

12. Gafni, E., Lamport, L.: Disk Paxos. Distrib. Comput. 1(16), 1$20(2003)$

13. Guerraoui, R.: Indulgent algorithms. In: Proceedings of the 19th Annual ACM Symposium on Principles of Distributed Computing (PODC) (2000)

14. Guerraoui, R., Herlihy, M., Kapałka, M., Pochon, B.: Robust contention management in software transactional memory. In: Proceedings of the Workshop on Synchronization and Concurrency in Object-Oriented Languages (SCOOL); in conjunction with the ACM Conference on Object-Oriented Programming, Systems, Languages and Applications (OOPSLA) (2005)

15. Guerraoui, R., Herlihy, M., Pochon, B.: Polymorphic contention management. In: Proceedings of the 19th International Symposium on Distributed Computing (DISC). LNCS, pp. 303-323. Springer, Heidelberg (2005)

16. Guerraoui, R., Herlihy, M., Pochon, B.: Toward a theory of transactional contention managers. In: Proceedings of the 24th Annual ACM Symposium on Principles of Distributed Computing (PODC) (2005)

17. Guerraoui, R., Schiper, A.: " $\Gamma$-accurate" failure detectors. In: Proceedings of the 10th International Workshop on Distributed Algorithms (WDAG). Springer, Heidelberg (1996)
18. Herlihy, M.: Wait-free synchronization. ACM Trans. Program. Lang. Syst. 13(1), 124-149 (1991)

19. Herlihy, M., Luchangco, V., Moir, M., Scherer III, W.N.: Software transactional memory for dynamic-sized data structures. In: Proceedings of the 22nd Annual ACM Symposium on Principles of Distributed Computing (PODC), pp. 92-101 (2003)

20. Herlihy, M., Luchango, V., Moir, M.: Obstruction-free synchronization: Double-ended queues as an example. In: Proceedings of the 23rd IEEE International Conference on Distributed Computing Systems (ICDCS), pp. 522-529 (2003)

21. Herlihy, M., Wing, J.M.: Linearizability: a correctness condition for concurrent objects. ACM Trans. Program. Lang. Syst. 12(3), 463-492 (1990)

22. Jayanti, P.: Robust wait-free hierarchies. J. ACM 44(4), 592614 (1997)

23. LaMarca, A.: A performance evaluation of lock-free synchronization protocols. In: Proceedings of the 13th Annual ACM Symposium on Principles of Distributed Computing (PODC), pp. 130-140 (1994)

24. Lamport, L.: The part-time parliament. ACM Trans. Comput. Syst. 16(2), 133-169 (1998)

25. Larrea, M., Fernández, A., Arévalo, S.: On the implementation of unreliable failure detectors in partially synchronous systems. IEEE Trans. Comput. 53(7), 815-828 (2004)

26. Moir, M., Anderson, J.H.: Wait-free algorithms for fast, long-lived renaming. Sci. Comput. Program. 25 (1995)

27. Scherer III, W.N., Scott, M.L.: Contention management in dynamic software transactional memory. In: Proceedings of the Workshop on Concurrency and Synchronization in Java Programs; in conjunction with the 23th Annual ACM Symposium on Principles of Distributed Computing (PODC) (2004)

28. Scherer III, W.N., Scott, M.L.: Advanced contention management for dynamic software transactional memory. In: Proceedings of the 24th Annual ACM Symposium on Principles of Distributed Computing (PODC) (2005) 